\title{
Model structure on the universe of all types in interval type theory ${ }^{\dagger}$
}

\author{
Simon Boulier and Nicolas Tabareau* (D) \\ Inria, France \\ *Corresponding author. Email: nicolas.tabareau@inria.fr
}

(Received 28 December 2018; revised 25 August 2020; accepted 25 August 2020; first published online 14 October 2020)

\begin{abstract}
Model categories constitute the major context for doing homotopy theory. More recently, homotopy type theory (HoTT) has been introduced as a context for doing syntactic homotopy theory. In this paper, we show that a slight generalization of HoTT, called interval type theory ( $(\mathrm{TTT})$, allows to define a model structure on the universe of all types, which, through the model interpretation, corresponds to defining a model structure on the category of cubical sets. This work generalizes previous works of Gambino, Garner, and Lumsdaine from the universe of fibrant types to the universe of all types. Our definition of $\square T T$ comes from the work of Orton and Pitts to define a syntactic approximation of the internal language of the category of cubical sets. In this paper, we extend the work of Orton and Pitts by introducing the notion of degenerate fibrancy, which allows to define a fibrant replacement, at the heart of the model structure on the universe of all types. All our definitions and propositions have been formalized using the Coq proof assistant.
\end{abstract}

Keywords: Homotopy type theory, model structure, fibrant types, interval type theory

\section{Introduction}

Homotopy type theory (HoTT) can be seen both as a language to formalize mathematics and as a language to do synthetic homotopy theory. Synthetic homotopy theory consists in proving homotopy properties in a syntactic language, here a type theory, which can then be interpreted in several models of homotopy theory (e.g. simplicial sets, cubical sets).

The first model of HoTT has been proposed by Voevodsky using a standard notion in homotopy theory - simplicial sets. This model has later been reworked and polished by Kapulkin and Lumsdaine (2012). In this model, a type is interpreted by a Kan simplicial set ${ }^{1}$ and univalent equality is interpreted by paths in those topological spaces.

The simplicial model uses classical logic crucially as it requires to decide whether a map is a degeneracy or not (Bezem et al. 2015). Cubical model, in which a type is interpreted by a Kan cubical set, have been considered to build an intuitionistic model of HoTT. There are several variants of this model, depending on which cube category is chosen:

- for cubes without connections, it is called the $\mathrm{BCH}$ model (from the initials of its authors) (Bezem et al. 2013, 2019)

- for cubes with connections, it is called the CCHM model (Cohen et al. 2017; Coquand et al. 2018).

- cartesian cube category (including diagonals) also give rise to a model of HoTT (Angiuli et al. 2018, 2019).

\footnotetext{
${ }^{\dagger}$ This work has been supported by the CoqHoTT ERC Grant 637339.
} 
In their works about CCHM, Coquand et al. not only develop a model of HoTT but also an internal language of this model to carry syntactically some constructions of the model. This internal language has been coined cubical type theory.

Recently, Orton and Pitts (2018a) have developed a new abstraction layer for the CCHM model. As Martin-Löf type theory can be interpreted in any presheaf category (and even any topos), they identified nine axioms which are valid through the interpretation in the CCHM model and which are enough to carry several constructions of the model (basically, all but the universe of fibrant types) and thus to define cubical type theory internally. So their type theoretic setting can be used to describe the CCHM model.

Orton and Pitts work in the internal language of the presheaf model considered. In this article, we call interval type theory (घTT), Martin-Löf type theory enriched with the constructions introduced by Orton and Pitts. Thus, 口TT can be seen as a syntactic subset of the internal language. It is a subset because all its constructions have their interpretation in the model but it is not complete. For instance, we use an intensional type theory while the internal language is extensional.

The simplicial and the cubical models have been settled to describe a notion of equality upto homotopy, satisfying the univalence axiom. But both models allow to interpret a second notion of equality, induced by the equality of the meta-theory. Voevodsky (2013) was the first to propose a type theory where this equality is reflected in the system and called it homotopy type system. In this system, the equality coming from the equality of the meta-theory is called strict, it enjoys uniqueness of identity proof (UIP), by opposition to the univalent equality which satisfies the univalence axiom. To avoid the collapse of those two equalities, and thus the inconsistency of the theory, the property of being fibrant (or Kan) is also reflected at the level of types. The idea is that the univalent equality can only be eliminated over a fibrant predicate, whereas the strict equality can be eliminated over any predicate. Later, Altenkirch et al. gave a more general analysis of a type theory with two equalities and a fibrancy mechanism and called it two-level type theory (Altenkirch et al. 2016; Capriotti 2017). In $\square \mathrm{TT}$, the strict equality is primitive and the univalent equality can be defined internally.

The main contribution of this paper is to show internally in पTT that there is a pre-model structure on the universe of all types. Through the interpretation in the model, a direct corollary of this internal construction is that the category of cubical sets is a model category.

A model category is a setting for homotopy theory. It consists of a category equipped with three classes of arrows - fibrations, cofibrations, and weak equivalences - enjoying several properties. In particular, they have to give rise to two ways of factorizing an arbitrary function. Fibrations can be seen as "nice surjections," cofibrations as "nice injections" and weak equivalence as "homotopy equivalences." Typical examples of model categories are Top, the category of topological spaces, sSet, the category of simplicial sets, and cSet, the category of cubical sets. Model categories are of great importance to compare those different settings in which to formalize homotopy theory, using the notion of Quillen equivalences.

It has already been shown in HoTT that there is a pre-model structure on the universe of fibrant types. The first factorization system (acyclic cofibrations and fibrations, or simply (AC,F)) has been given by Gambino and Garner (2008) using homotopy fibers and the second factorization system (cofibrations and acyclic fibrations or simply (C,AF)) has been given by Lumsdaine (2011) using mapping cylinders. HoTT can be seen as a fibrant fragment of $\square \mathrm{TT}$, this result can thus be understood as a pre-model structure on the universe $\mathscr{U} \mathscr{F}$ of fibrant types. Our work is thus a generalization to the universe $\mathscr{U}$ of arbitrary types. $\mathscr{U} \mathscr{F}$ corresponds to the homotopy category thus its model structure is quite trivial as everything in the universe it already fibrant. To the opposite, $\mathscr{U}$ corresponds to the general model category which has a much more complex model structure. In particular, our construction requires the existence of a fibrant replacement, which is not needed in the fibrant case as all types are fibrant.

Note that the notion of fibrant replacement is not admissible with the standard notion of fibrancy provided by a two-level type theory. For instance, it is inconsistent with the existence 
of types which are not h-sets (Capriotti 2017) or with an interval (Homotopy Type Theory wiki 2014). Thus, another contribution of this paper is to distinguish between two notions of fibrancy degenerate and regular - which allows to define a notion of fibrant replacement compatible with the substitution of type theory.

A description of a model structure on the category of cubical sets has recently been given by Sattler (2017) and it should be interesting to compare its model structure with the one given by the interpretation of our internal model structure in QTT in the cubical sets model.

Plan of the paper. In Sections 2 and 3, we make precise our notion of $\square \mathrm{TT}$ and redefine a whole part (paths, identity types) of the CCHM model in 『TT as there are still few references on the topic. We also introduce a new weaker notion of fibrancy called degenerate fibrancy. In Section 4, we discuss briefly the model of 口TT and look at the induced notion of fibrancy in cubical sets through the model interpretation. In Section 5, we construct the fibrant replacement as a quotient inductive type (QIT) in पTT. Section 6 provides the complete definition of a model category. Then, we describe internally in $\mathrm{QTT}$ the (AC,F) weak factorization system (Section 7) and two variants of the (C,AF) weak factorization system (Section 9), one which makes use of the notion of mapping cylinders (Section 8), and one which makes us of the notion of partial elements. Finally, we provide a complete description of the model structure on $\mathscr{U}$ in $\square \mathrm{TT}$ (Section 10) and discuss the formalization (Section 11).

Formalization. Following Orton and Pitts approach, all the results of our article have been formalized using an axiomatic presentation of एTT. The formalization as been carried in Coq (while Orton and Pitts used Agda) and can be found in the following repository:

https:/gitlab.inria.fr/sboulier/thesis-formalizations/blob/emptyctx/InternalCubical-Coq

For each subsection, we give a direct link to the corresponding file in the repository.

\section{Interval Type Theory}

The kernel of $\square T$ T is Martin-Löf type theory with $\Pi$ types, $\Sigma$ types, an impredicative universe of propositions $\mathbb{P}$ and a strict equality $\equiv$ modeled using an identity type. Its typing rules are given in Figures 1 and 2 . We call the equality strict because it enjoys UIPs. The type of strict equalities live in the universe of propositions $\mathbb{P}$. As a consequence, UIP follows from the more general principle of proof irrelevance. We also suppose that the strict equality enjoys function extensionality and propositional extensionality. The conversion encompasses $\beta$ reduction for functions, pairs, and equality, and $\eta$ reduction for functions and pairs. It is written as $\simeq_{\beta \eta}$.

The impredicative ${ }^{2}$ universe $\mathbb{P}$ is closed under $\Pi$ types (written $\forall$ ), $\Sigma$ types (written $\exists$ ), disjunction $\vee$, unit type $T$, and empty type $\perp$. The complete rules are given in Appendix A. In $\square T T$, we will also consider a universe $\mathscr{U}$, closed under $\Pi$ and $\Sigma$ types. The $E l$ operator on those two universes, which turns a proposition or a code $A$ into a type, is written $\underline{A}$. We also consider that $\mathscr{U}$ contains a unit type $\mathbb{1}$ and a type of booleans (which will be noted OI in this paper) with their standard introduction and elimination rules.

We use the usual notations: $A \rightarrow B$ for non-dependent $\Pi / \forall$ type; $A \times B$ for non-dependent $\Sigma$ type; $A \leftrightarrow B$ for $(A \rightarrow B) \times(B \rightarrow A) ; P \Leftrightarrow Q$ for its counterpart in $\mathbb{P} ; p \#^{P} t$ for the transport along a strict equality (non-dependent version of $J_{\equiv}$ ). We say that $P$ is a type family over a type $A$ if it is a type in a context ending by $A: \Gamma, x: A \vdash P$. We will write $x: A$. $P$ such a family. If $P$ fits in an universe $\mathscr{U}$, it amounts to having a function $A \rightarrow \mathscr{U}$. To ease the reading, many arguments of functions will be considered as implicit arguments and thus be omitted in the paper, as well as the underlines of the $E l$ operator which can be inferred easily. Last, the context $\Gamma$, always supposed well formed, is often omitted in the typing rules. An omitted context is written $\vdash$, while an empty context is written $\emptyset \vdash$. 


$$
\begin{aligned}
& \overline{\emptyset \vdash} \quad \frac{\Gamma \vdash A}{\Gamma, x: A \vdash} \quad \frac{\Gamma \vdash x: A \in \Gamma}{\Gamma \vdash x: A} \quad \frac{\Gamma \vdash t: A \quad \Gamma \vdash B \quad A \simeq_{\beta \eta} B}{\Gamma \vdash t: B} \\
& \frac{\Gamma \vdash A \quad \Gamma, x: A \vdash B}{\Gamma \vdash \Pi x: A \cdot B} \quad \frac{\Gamma, x: A \vdash t: B}{\Gamma \vdash \lambda x: A \cdot t: \Pi x: A \cdot B} \quad \frac{\Gamma \vdash t: \Pi x: A \cdot B \quad \Gamma \vdash u: A}{\Gamma \vdash t u: B\{x:=u\}} \\
& \frac{\Gamma \vdash A \quad \Gamma, x: A \vdash B}{\Gamma \vdash \Sigma x: A . B} \quad \frac{\Gamma \vdash t: A \quad \Gamma \vdash u: B\{x:=t\}}{\Gamma \vdash(t, u): \Sigma x: A \cdot B} \quad \frac{\Gamma \vdash t: \Sigma x: A \cdot B}{\Gamma \vdash \pi_{1}(t): A} \\
& \frac{\Gamma \vdash t: \Sigma x: A \cdot B}{\Gamma \vdash \pi_{2}(t): B\left\{x:=\pi_{1}(t)\right\}} \quad \frac{\Gamma \vdash}{\Gamma \vdash \mathbb{P}} \quad \frac{\Gamma \vdash A: \mathbb{P}}{\Gamma \vdash \underline{A}} \quad \frac{\Gamma \vdash}{\Gamma \vdash \mathscr{U}} \quad \frac{\Gamma \vdash A: \mathscr{U}}{\Gamma \vdash \underline{A}} \\
& \frac{\Gamma \vdash t: A \quad \Gamma \vdash u: A}{\Gamma \vdash t \equiv_{A} u: \mathbb{P}} \quad \frac{\Gamma \vdash t: A}{\Gamma \vdash \mathbf{r e f l}_{\equiv} t: \underline{t \equiv_{A} t}} \\
& \frac{\Gamma, x: A, p: t \equiv_{A} x \vdash P \quad \Gamma \vdash q: t \equiv_{A} t^{\prime} \quad \Gamma \vdash u: P\left\{x:=t, p:=\mathbf{r e f l}_{\equiv} t\right\}}{\Gamma \vdash J_{\equiv}(P, q, u): P\left\{x:=t^{\prime}, p:=q\right\}} \\
& (\lambda x: A . t) u \simeq_{\beta \eta} t\{x:=u\} \quad \lambda x: A \cdot t x \simeq_{\beta \eta} t \\
& \pi_{1}\left(t, t^{\prime}\right) \simeq_{\beta \eta} t \quad \pi_{2}\left(t, t^{\prime}\right) \simeq_{\beta \eta} t^{\prime} \quad\left(\pi_{1}(t), \pi_{2}(t)\right) \simeq_{\beta \eta} t \\
& J_{\equiv}(P, \text { refl } \equiv t, u) \simeq_{\beta \eta} u
\end{aligned}
$$

Figure 1. Martin-Löf type theory.

$$
\begin{gathered}
\frac{\Gamma \vdash A: \mathbb{P} \quad \Gamma \vdash u, v: \underline{A}}{\Gamma \vdash \operatorname{proofIrr}_{A}(u, v): \underline{u \underline{A}_{\underline{A}}}} \quad \frac{\Gamma \vdash f, g: \Pi x: A \cdot B \quad \Gamma \vdash e: \Pi x: A . \underline{f x \equiv_{B} g x}}{\Gamma \vdash \operatorname{funext}(e): \underline{f \equiv_{\Pi A B} g}} \\
\frac{\Gamma \vdash P, Q: \mathbb{P} \quad \Gamma \vdash e: \underline{P \Leftrightarrow Q}}{\Gamma \vdash \operatorname{propext}(e): \underline{P \equiv_{\mathbb{P} Q}}}
\end{gathered}
$$

Figure 2. Axioms for strict equality in Martin-Löf type theory.

In Sections 5 and 8, we will use some QITs. QITs are a generalization of inductive types where equality between elements of the inductive type can be defined at the same time as the definition of its constructors. They are the strict counterpart of higher inductive types (HITs) for a strict equality (i.e. enjoying UIP). HITs and QITs form an active field of research and both their syntax and their semantic are not fully established. We will use an intuitive syntax and will give the introduction and elimination rules for each one. See Coquand et al. (2018) and Cavallo and Harper (2019) for recent developments on HITs and Altenkirch et al. (2018) for QITs.

In this paper, we work in 口TT which is a syntactic approximation of the internal language of the CCHM model. It consists of the above-mentioned Martin-Löf type theory enriched with:

- an interval ₫

- a universe of cofibrant propositions Cof

satisfying nine axioms highlighted by Orton and Pitts (2018a) which we now detail.

\subsection{Interval [Interval.v]}

The interval $\square$ is the first distinguishing feature of $\square T$ T. It is a closed type which has two endpoints 0 and 1 and supports $\min (\sqcap)$ and $\max (\sqcup)$ operations:

$$
\vdash \square \quad \vdash 0,1: \rrbracket \quad \vdash \sqcap: \square \rightarrow \square \rightarrow \square \quad \vdash \sqcup: \square \rightarrow \square \rightarrow \square
$$




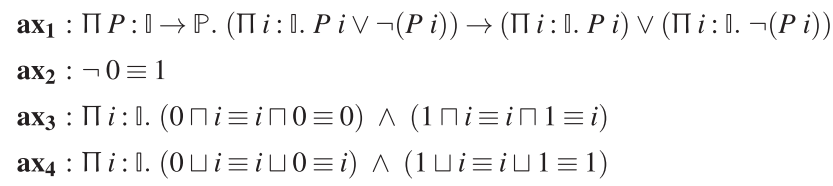

Figure 3. Axioms satisfied by the interval 0 .

$\square$ is required to satisfy the four axioms given in Figure 3. The axioms ensure that the interval is connected $\left(\mathbf{a x}_{1}\right)$ that the two endpoints are distinct for strict equality $\left(\mathbf{a x}_{2}\right)$ and that $\min$ and $\max$ give a path algebra structure to $\square\left(\mathbf{a x}_{\mathbf{3}}\right.$ and $\left.\mathbf{a x}_{\mathbf{4}}\right)$. A path algebra structure is like a bounded lattice but without associativity, commutativity, and absorption law.

An important difference with the cubical model CCHM is that the interval does not have negation not $: \square \rightarrow \square$. We could add it, restricting slightly the presheaf models in which we can interpret our theory. But here, we stick to Orton and Pitts approach.

A variable typed by the interval is called dimension and having a dimension $i: \square$ in the context corresponds to going one dimension up. An element of a type $a: A$ represents a point:

A function $p: \square \rightarrow A$ represents a line in $A$, also called a path, between the two points $p 0$ and $p 1$ :
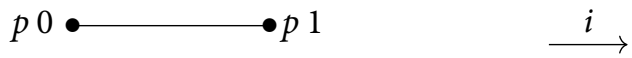

The constant function $\lambda_{-}:$: $a$ is the constant path from $a$ to $a$. It is written idpath $a$.

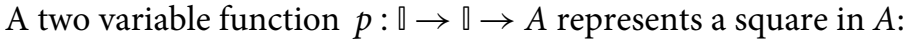
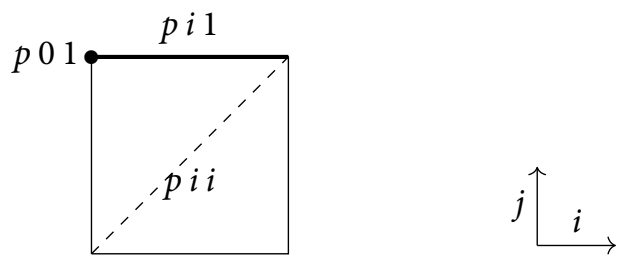

For instance, the up left point is $p 01$, the upper side is the line $\lambda i$. $p i 1$, and the upward diagonal is $\lambda i$. $p i$. Note that it is not possible to represent the other diagonal because there is no negation operation.

As a last example, a function $p: \llbracket \rightarrow A$ is a line between $a$ and $b$ :

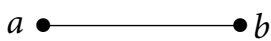

Then $\lambda i, j . p(i \sqcup j)$ is the following square:
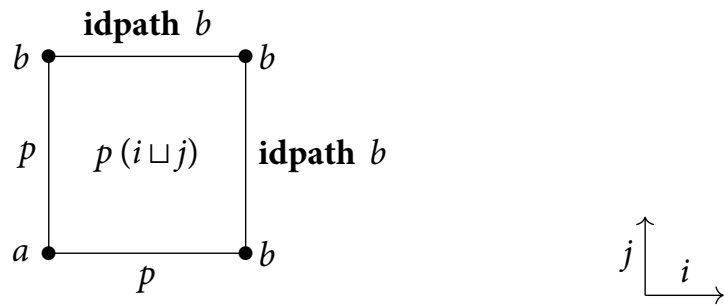


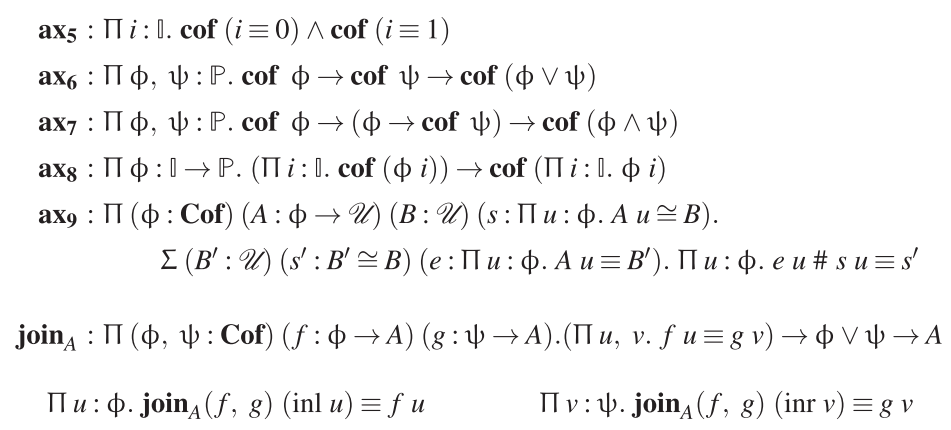

Figure 4. Axioms satisfied by Cof.

\subsection{Cofibrant propositions [Cof.v]}

The other important feature of घTT is the universe of cofibrant propositions Cof. It is a subtype of $\mathbb{P}$ defined by a predicate cof asserting that a proposition is cofibrant, and it satisfies the axioms of Figure 4:

$$
\vdash \operatorname{cof}: \mathbb{P} \rightarrow \mathbb{P} \quad \text { Cof }:=\Sigma P: \mathbb{P} \text {. cof } P
$$

Cofibrant propositions represent formulas restricting the introduced dimensions. A typical cofibrant proposition is for instance:

$$
(i \equiv 0) \vee((i \equiv 1) \wedge(j \equiv 0))
$$

We have seen that a function $p: \llbracket \rightarrow \square \rightarrow A$ is a square. Now, a function restricted by this formula:

$$
p^{\prime}: \Pi i, j: \text { 『. }(i \equiv 0) \vee((i \equiv 1) \wedge(j \equiv 0)) \rightarrow A
$$

represents the following subsquare:
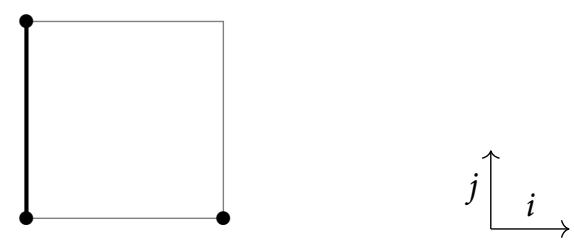

An element of a type $A$ specified only on a face $\phi(f: \phi \rightarrow A)$ is called a partial element of type $A$.

The axioms assert that Cof contains endpoint equality $\left(\mathbf{a x}_{\mathbf{5}}\right)$ is closed under disjunction, dependent conjunction, and universal quantification over $\square\left(\mathbf{a x}_{\mathbf{6}}, \mathbf{a x}_{7}\right.$, and $\left.\mathbf{a x}_{\mathbf{8}}\right)$.

The last axiom is a strictness axioms asserting that under some conditions, a type can be replaced by a strictly equivalent type satisfying additional strict equalities. By strict equivalence, we mean a type equivalence for the strict equality:

$$
A \cong B:=\Sigma(f: A \rightarrow B)(g: B \rightarrow A) .(g \circ f \equiv \mathbf{i d}) \wedge(f \circ g \equiv \mathbf{i d}) .
$$

Axiom 5 together with propositional extensionality ensures that true and false proposition $\top$ and $\perp$ are cofibrant. For $\phi$ and $\psi$ in Cof, we will continue writing $\phi \wedge \psi$ for the element of Cof associated with the proposition $\pi_{1}(\phi) \wedge \pi_{1}(\psi)$. The same for $\phi \vee \psi, i \equiv 0, \perp, \ldots$ We will also blithely write $\phi$ instead of $\pi_{1}(\phi)$.

As last requirement on Cof, we suppose that the universe of cofibrant propositions allows to form the join of two partial elements. Given two cofibrant propositions $\phi, \psi$ : Cof and two partial elements $f$ and $g$ which agree where there are both defined:

$$
f: \phi \rightarrow A \quad \quad g: \psi \rightarrow A \quad \Pi(u: \phi)(v: \psi) . f u \equiv g v
$$


the join of $f$ and $g$ is a partial element on $\phi \vee \psi$ :

$$
\operatorname{join}_{A}(f, g): \phi \vee \psi \rightarrow A
$$

such that $\operatorname{join}_{A}(f, g)($ inl $u) \equiv f u$ and $\operatorname{join}_{A}(f, g)$ (inr $\left.v\right) \equiv g v$. The join cannot be defined by usual disjunction because this would require $\vee$-elimination to an arbitrary type while it is restricted to $\mathbb{P}$.

\section{Paths and Fibrancy}

In the previous section, we have specified what is पTT. We can now describe some basic and fundamental constructions of HoTT directly inside 『TT.

\subsection{Paths [Paths.v]}

The first fundamental construction that can be carried over internally in ITT is the notion of paths. A path between two points is a line with these points at its ends. We write $a \sim_{A} b$ the type of paths between $a$ and $b$ in $A$. Reflexivity is given by a constant path:

$$
\begin{aligned}
a \sim_{A} b:= & \Sigma p: \llbracket \rightarrow A \cdot p 0 \equiv_{A} a \wedge p 1 \equiv_{A} b \\
\text { idpath }_{A} a & : a \sim_{A} a \\
& :=\left(\lambda_{-}: \square . a, \text { refl } \equiv a, \text { refl } \equiv a\right)
\end{aligned}
$$

Path types are not directly the types by which we will interpret the univalent equality because the transport is not strictly constant on identity paths. Instead, we will use identity types, which are built on top of them (Section 3.4).

The fact that paths are functions has nice consequences. For instance, function extensionality for paths becomes trivial: it is only a change in the order of the arguments of the path seen as a function. If $p$ is a proof of type $\Pi x: A . f x \sim_{B} g x$, then $\lambda i . \lambda x: A . p x i$ is a path between $f$ and $g$.

In the same spirit, paths enjoy contractibility of singletons. A type is contractible if it is inhabited and if all its elements are path-equal:

$$
\text { Contr } A:=\Sigma a: A . \Pi x: A . a \sim_{A} x
$$

For every type $A$ and $x: A$, the type of singletons in $x$ is contractible:

$$
\operatorname{Contr}\left(\Sigma y: A . x \sim_{A} y\right)
$$

The center of contraction is $\left(x, \mathbf{i d p a t h}_{A} x\right)$ and the path between $\left(x, \mathbf{i d p a t h}_{A} x\right)$ and $(y, p)$ is given by:

$$
\lambda i .(p i, \lambda j \cdot p(i \sqcap j))
$$

\subsection{Fibrancy [Fibrations.v]}

Now that we have path types, we want to transport along them. Transport is only valid along fibrant type families so we have to define fibrancy.

As we said, a partial elements of a type $A$ is an element of $A$ only specified on a restricted face of the current context. We write $\square A$ the type of partial elements of $A$ :

$$
\square A:=\Sigma \phi: \text { Cof. } \phi \rightarrow A
$$

Let $a$ : $A$ be an element of $A$ and $(\phi, u)$ be a partial element, we say that $a$ extends $(\phi, u)$ if they coincide where $u$ is defined:

$$
(\phi, u) \nearrow a:=\Pi w: \phi \cdot u w \equiv_{A} a
$$


There is always the empty inhabitant of $\square A$ :

$$
(\perp, \perp-\text { elim) : } \square A
$$

It is the partial element of $A$ which is specified nowhere. Every partial element $(\phi, f)$ of $A$ turns out to be path-equal to this empty inhabitant via the path $\lambda i .\left(\phi \wedge i \equiv 1, f \circ \pi_{1}\right)$. Hence, the type $\square A$ is always contractible:

\section{$\operatorname{Contr}(\square A)$}

A type has an extension structure if all its partial elements can be extended:

$$
\text { Ext } A:=\Pi a: \square A . \Sigma a_{1}: A . a \nearrow a_{1}
$$

Fibrancy is a weaker condition than having an extension structure where the partial element is required to be specified in at least one endpoint.

To overcome the lack of negation, Orton and Pitts parameterize their definition of fibrancy by the direction in which the extension is done. To do this, we introduce the inductive type OI which has two elements $\mathrm{O}$ and $\mathrm{I}$ (it is thus a synonym for bool):

$$
O I:=0 \mid \mathrm{I}
$$

And we define $\iota: O I \rightarrow \square$, the coercion to the interval, by $\iota 0:=0$ and $\iota \mathrm{I}:=1$. In the following, we will suppose that this coercion is always implicitly inserted where needed. We will also use the negation !: OI $\rightarrow$ OI which is defined by:

$$
\begin{aligned}
& ! O:=I \\
& ! \mathrm{I}:=0
\end{aligned}
$$

Definition 1. A type $A$ is said to be fibrant, if the following type is inhabited:

$$
\text { Fib } A:=\Pi(e: \text { OI })(\phi: \text { Cof })(a: \Pi i: \square . \phi \vee i \equiv e \rightarrow A) . \Sigma a_{1}: A .(\phi, a ! e \circ \text { inl }) \nearrow a_{1}
$$

It means that every partial element specified at least in $i \equiv$ e can be extended.

This type is not exactly the one proposed by Orton and Pitts but is strictly equivalent. For the sake of completeness, they use

$$
\Pi(e: \text { OI })(\phi: \text { Cof })(a: \rrbracket \rightarrow \phi \rightarrow A) .\left(\Sigma a_{0}: A .(\phi, a e) \nearrow a_{0}\right) \rightarrow\left(\Sigma a_{1}: A .(\phi, a ! e) \nearrow a_{1}\right)
$$

Our variant is slightly more compact and will allow to define the fibrant replacement in a simpler way.

Fibrancy is weaker than having an extension structure:

$$
\text { Ext } A \rightarrow \text { Fib } A
$$

In fact, it turns out that a type has an extension structure if and only if it is both fibrant and contractible.

Proposition 1. For any type A we have

$$
\text { Ext } A \leftrightarrow(\text { Fib } A \times \operatorname{Contr} A)
$$

It is not a strict equivalence a priori.

Fibrancy generalizes to type families in the following way.

Definition 2. A type family $x: A$. P is said to be regularly fibrant ${ }^{3}$ if the following type is inhabited:

$$
\begin{gathered}
\operatorname{RFib}(x . P):=\Pi(e: \text { OI })(a: \llbracket \rightarrow A)(\phi: \operatorname{Cof})(p: \Pi i: \llbracket . \phi \vee i \equiv e \rightarrow P\{x:=a i\}) . \\
\Sigma p_{1}: P\{x:=a ! e\} .(\phi, p ! e \circ \text { inl }) \nearrow p_{1}
\end{gathered}
$$


The operation to which fibrancy gives access is called composition. If $P$ is regularly fibrant, then we write

$$
\operatorname{comp}_{P}(e: \text { OI })(a: \rrbracket \rightarrow A)(\phi: \text { Cof })(p: \Pi i: \rrbracket . \phi \vee i \equiv e \rightarrow P\{x:=a i\}): P\{x:=a ! e\}
$$

And for all $w: \phi$ we have that:

$$
\operatorname{comp}_{P} \text { e a } \phi p \equiv p ! e(\text { inl } w)
$$

A type A amounts to be fibrant if and only if the constant family over unit _: $\mathbb{1}$. A is regularly fibrant.

For a type family $x: A . P$, it is not equivalent to be regularly fibrant and that for every $x, P$ is fibrant. The later is weaker than the former. We will say that a type family is degenerately fibrant ${ }^{4}$ or pointwise fibrant if we only have the weaker condition:

$$
\operatorname{DFib}(x . P):=\Pi x: A \text {. Fib } P
$$

Remark 2. A notable exception is the case when $P$ has all its fibers contractible in which case regular fibrancy and uniform fibrancy coincide. Indeed, by Proposition 1, we have

$$
\operatorname{DFib}(x . P) \times(\Pi x \text {. Contr } P) \leftrightarrow \Pi x \text {. Ext } P
$$

But the extension structure on fibers is enough to establish the regular fibrancy, hence we also have

$$
\operatorname{DFib}(x . P) \times(\Pi x . \text { Contr } P) \leftrightarrow \operatorname{RFib}(x . P) \times(\Pi x \text {. Contr } P) .
$$

For a two variables type family $x: A, y: B . P$, we will write $\mathbf{R F i b}_{2}(x, y . P)$ if the type family over the sigma $\Sigma x: A . B$ is regularly fibrant (and so one for three variables families):

$$
\operatorname{RFib}_{2}(x: A, y: B . P):=\operatorname{RFib}\left(z . P\left\{x:=\pi_{1}(z), y:=\pi_{2}(z)\right\}\right)
$$

A useful remark is that regular fibrancy is stable under precomposition:

$$
\boldsymbol{\operatorname { R F i b }}(y . P) \rightarrow \operatorname{RFib}(x . P\{y:=f x\})
$$

Orton and Pitts proved the following propositions, which show that the category with families of regularly fibrant families supports several type formers.

\section{Proposition 3.}

$$
\text { Fib } \mathbb{1} \text { and Fib } \mathbb{N}
$$

Proposition 4. For $x: X$. $A$ and $x: X, a: A . B$, if $\mathbf{R F i b}(x . A)$ and $\mathbf{R F i b}_{2}(x, a . B)$ then,

$$
\begin{aligned}
& \operatorname{RFib}(x: X . \Pi a: A . B) \\
& \operatorname{RFib}(x: X . \Sigma a: A . B)
\end{aligned}
$$

Proposition 5. For $x: X$. A, if $\mathbf{R F i b}(x . A)$, then path types on A are regularly fibrant as a three variables family:

$$
\operatorname{RFib}_{3}\left(x: X, a: A, b: A . a \sim_{A} b\right)
$$

See Orton and Pitts' article or the formalization for the proofs.

We also have that the universe of pretypes is fibrant. In fact, it even has an extension structure. This is quite surprising because it means that for all types $A$ and $B$, there is a path connecting $A$ and $B$ :

$$
A \sim \mathscr{U}_{B} B
$$

However, this will not be enough to transport along it and get a map $A \rightarrow B$ because the type family $X: \mathscr{U} . \underline{X}$ is not regularly fibrant. 
Proposition 6. The universe has an extension structure, and hence is fibrant:

\section{Ext $\mathscr{U}$}

Proof. Let $A: \phi \rightarrow \mathscr{U}$ be a partial element of $\mathscr{U}$. We have to give $B^{\prime}: \mathscr{U}$ such that for all $w: \phi$, $A w \equiv B^{\prime}$.

$B^{\prime}$ is given by the strictness axiom taken in $A$. There are two choices for the $B$ appearing in the axiom: either $\Pi w: \phi . A w$ or $\Sigma w: \phi . A w$, both work.

From the composition operation, we can derive a richer operation: Kan filling.

Proposition 7. If $x: A$. $P$ is regularly fibrant, then we can define an operation:

$$
\text { fill }_{P}(e: 0 \mathrm{I})(a: \square \rightarrow A)(\phi: \text { Cof })(p: \Pi i: \square . \phi \vee i \equiv e \rightarrow P\{x:=a i\})(i: \square): P\{x:=a i\}
$$

such that for all $e, \phi, a$, and $p$, fill $p$ agrees with $p$ on $\phi \vee i \equiv e$ and with comp in !e:

$$
\begin{gathered}
\Pi i: \text { 『. } w \phi \vee i \equiv e \text { fill }{ }_{P} \text { e a } \phi p i \equiv p i w \\
\quad \text { fill }_{P} \text { e a } \phi p ! e \equiv \operatorname{comp}_{P} \text { e a } \phi p
\end{gathered}
$$

This is a notable feature of the CCHM model that Kan filling can be defined from composition, it is not the case in $\mathrm{BCH}$ for instance. Again, see Orton and Pitts article or the formalization for the proof.

\subsection{Transport [Paths. v]}

Now that if we have defined fibrancy, we can at last define transport along a path.

Proposition 8. Let $x: A$. $P$ be a regularly fibrant type family, $p: a \sim_{A} b$ a path between two points of $A$ and $u$ of type $P a$. Then, $u$ can be transported along $p$ to get an element of type $P b$ :

$$
\operatorname{transport}(P, p, u): P b
$$

Proof. The transport is given by:

$$
\operatorname{comp}_{P} 0 \perp \perp-\operatorname{elim} u
$$

modulo rewriting by strict equalities.

Transport together with contractibility of singletons give the dependent elimination for path types (still restricted to regularly fibrant families).

However, paths have a defect: the transport does not compute on reflexivity. For all $a: A$ and $u: P a$, we have a path:

$$
\operatorname{transport}\left(P, \text { idpath }_{A} a, u\right) \sim_{P a} u
$$

but this equality does not hold strictly a priori. Identity types are introduced in the next section to remedy this.

With the transport and the dependent elimination, we can recover all the groupoidal laws satisfied by a univalent equality. First of them are inverse and concatenation:

$$
\begin{gathered}
-^{-1}: x \sim_{A} y \rightarrow y \sim_{A} x \\
-_{-} \cdot: x \sim_{A} y \rightarrow y \sim_{A} z \rightarrow x \sim_{A} z
\end{gathered}
$$

There are some operations which are proven with a transport in HoTT setting but which does not need the fibrancy structure in the cubical setting. They can be defined directly using the cubical definition of paths. For instance:

$$
\begin{gathered}
\mathbf{a p}_{f}: x \sim_{A} y \rightarrow f x \sim_{B} f y \\
\operatorname{ap10}_{x}: f \sim_{A \rightarrow B} g \rightarrow f x \sim_{B} g x
\end{gathered}
$$


To a path $p$, the first associates the path $\lambda$ i.f $(p i)$ and the second the path $\lambda i$. ( $p i) x$. It is the same phenomenon that for the definition of function extensionality: as a path type is a richer type than in HoTT, some definitions are simpler.

\subsection{Identity types [Id.v]}

Identity types are a modified version of path types to recover the strict equality for the transport of reflexivity. They were introduced by Swan (2016) and reused in the CCHM model (Cohen et al. 2017). An element of an identity type is a path together with a cofibrant proposition indicating "where the path is refl." We will note $x={ }_{A} y$ the identity types:

$$
x={ }_{A} y:=\Sigma\left(p: x \sim_{A} y\right)(\phi: \text { Cof }) . \phi \rightarrow \Pi i: \text {. } p i \equiv_{A} x
$$

Reflexivity is given by the constant path with the proposition "true":

$$
\begin{aligned}
\operatorname{refl}_{=} x & : x={ }_{A} x \\
& :=\left(\text { idpath }_{A} x, \top, \lambda w i . \text { refl }_{\equiv} x\right)
\end{aligned}
$$

Identity types and paths are logically equivalent. From identity types to paths, there is a forgetful map, and in the other direction we use the proposition "false":

$$
\begin{aligned}
\text { id2paths } & : x=_{A} y \rightarrow x \sim_{A} y \\
& :=\lambda(p,-,) \cdot p \\
\text { paths2id } & : x \sim_{A} y \rightarrow x={ }_{A} y \\
& :=\lambda p \cdot(p, \perp, \perp-\text { elim })
\end{aligned}
$$

Note that id2paths sends "refl to refl" (i.e. refl= to idpath), but paths2id does not send idpath to refl $_{=}$.

Given the extra proposition $\phi$ asserting where the path is refl, it is possible to define a dependent

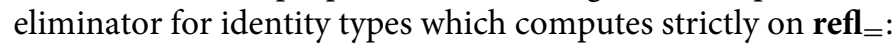

$$
\begin{aligned}
& x: A, p: t=_{A} x \vdash P \quad \vdash H: \operatorname{RFib}_{2}(x, p \cdot P) \\
& \frac{\vdash q: t={ }_{A} t^{\prime} \quad \vdash u: P\left\{x:=t, p:=\operatorname{refl}_{=} t\right\}}{\vdash J_{=}(P, H, q, u): P\left\{x:=t^{\prime}, p:=q\right\}} \quad J_{=}\left(P, H, \mathbf{r e f l}_{=} t, u\right) \equiv u
\end{aligned}
$$

\section{Models of $[T T$}

The intended model of घTT is the CCHM model, although a wider class of presheaf model can be considered, see Orton and Pitts (2018a).

In the presheaf model of dependent type theory, a context is interpreted by a presheaf which is a type parametrized by the objects of a category $\mathscr{C}$. Presheaves are used to model notions, such as "variation over time," "resource availability" and, in our case, "dimension." The presheaf model support $\Pi$ types, $\Sigma$ types, and universes. See Hofmann (1997) for an account of the presheaf model.

The CCHM model is a presheaf model over a cube category with connections, which will be

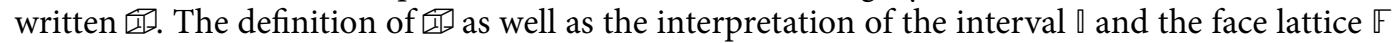
are given in Cohen et al. (2017, Section 8.1).

To interpret $\square \mathrm{TT}$, it remains to check that the presheaf model preserves function extensionality, propositional extensionality, proof irrelevance, and QITs. It is true for the three axioms. It should also be the case for QITs but we did not do the formal verification.

Remark. The requirement of having propositional extensionality in the meta-theory is very strong. It seems that the use of this axiom could be avoided by working with the face lattice $\mathbb{F}$ 
of CCHM instead of the cofibrancy predicate cof (the definition of Cof as $\Sigma P: \mathbb{P}$. cof $P$ has really a set theoretic flavor). We let this for future works.

\subsection{Degenerate fibrancy}

口TT can be viewed as a syntactic subset of the internal language of CCHM, meaning that the syntax of $\square T$ T can be used to carry constructions in CCHM. Indeed, in the presheaf model, no interpretation of rule makes the assumption that the premises of the rule are "coming from the syntax." For instance, the interpretation of the formation rule of $\Pi$ types can be carried for arbitrary presheaf families A over $\Gamma$ and $\mathrm{B}$ over $\Gamma, A$, even if they are not the interpretation of a type of $\square \mathrm{TT}$ :

$$
\frac{\Gamma \vdash A \quad \Gamma, A \vdash B}{\Gamma \vdash \Pi A B}
$$

Hence, for an arbitrary presheaf family $A$, we can form the presheaf family Fib $A$ by unfolding the definition of the Fib type former. Taking the proposition-as-types point of view, we say that the presheaf family $A$ is fibrant if the presheaf family Fib $A$ has a section. In this paragraph, we compare this induced notion of fibrancy with the one introduced in Coquand et al. article's.

Even if we do not want to detail here the definition of the cube category $⿴ 囗 ⿱ 一 一)$, we need to know a little about it. Its objects are written $I, J, \ldots$ and are finite sets of dimensions. For each object $I$, there exists a fresh dimension not in $I$ written $i$ \# $I$ giving rise to an object $I, i$ which is one dimension higher. There are two arrows $I \rightarrow I, i$ written $(i=0),(i=1)$ and called face maps. And in the other direction, there is an arrow $s_{I}: I, i \rightarrow I$ called a degeneracy. We have $s_{I}(i=0) \equiv s_{I}(i=1) \equiv \mathbf{i d}_{I}$.

Let us recall the definition of fibrancy introduced in Cohen et al. (2017) - we only rephrased it to use Orton and Pitts' trick to avoid negation.

Definition 3 (CCHM fibrancy). Let $\Gamma \vdash A$ be a presheaffamily. Then, $A$ is fibrant if it is equipped with a composition structure meaning that, for each $e: 0 I, I:$ 四, $i$ \# $I, \rho: \Gamma(I, i), \phi: \mathbb{F}(I)$, u partial element in $A \rho$ of extent $\phi, a_{0}: A(\rho(i=e))$ such that for all $f: J \rightarrow I$ with $\phi f \equiv 1_{\mathbb{F}}, a_{0} f \equiv u_{(i=e) f}$, we have an element:

$$
\operatorname{comp}\left(e, I, i, \rho, \phi, u, a_{0}\right): A(\rho(i=! e))
$$

such that for any $f: J \rightarrow I$ and $j \# J$,

$$
\left(\operatorname{comp}\left(e, I, i, \rho, \phi, u, a_{0}\right)\right) f \equiv \operatorname{comp}\left(e, J, j, \rho(f, i=j), \phi f, u(f, i=j), a_{0} f\right)
$$

and $\operatorname{comp}\left(e, I, i, \rho, 1_{\mathbb{F}}, u, a_{0}\right) \equiv u_{(i=! e)}$.

Now, if we unfold our definition of fibrancy, we get the following.

Proposition 9. Let $\Gamma \vdash A$ and $\Gamma, A \vdash P$ be presheaffamilies. Then, there is a section of $\Gamma \vdash$ RFib $P$ if and only if, for each $e: 0 \mathrm{I}, I:$ 四, $i$ \# $I, \rho: \Gamma(I), x: A\left(\rho s_{I}\right), \phi: \mathbb{F}(I)$, u partial element in $P\left(\rho s_{I}, x\right)$ of extent $\phi, p_{0}: P(\rho, x(i=e))$ such that for all $f: J \rightarrow I$ with $\phi f \equiv 1_{\mathbb{F}}, p_{0} f \equiv u_{(i=e) f}$, we have an element:

$$
\operatorname{comp}\left(e, I, i, \rho, x, \phi, u, p_{0}\right): P(\rho, x(i=! e))
$$

such that for any $f: J \rightarrow I$ and $j \# J$,

$$
\left(\operatorname{comp}\left(e, I, i, \rho, x, \phi, u, p_{0}\right)\right) f \equiv \operatorname{comp}\left(e, J, j, \rho f, x(f, i=j), \phi f, u(f, i=j), p_{0} f\right)
$$

and $\operatorname{comp}\left(e, I, i, \rho, x, 1_{\mathbb{F}}, u, p_{0}\right) \equiv u_{(i=! e)}$.

We remark the introduction of a degenerate part (the $\rho s_{I}$ ) and a regular part (the $x$ ). Thus, we will say that $P$ is regularly fibrant with respect to $A$ and degenerately fibrant with respect to $\Gamma$, or simply that $P$ is regularly fibrant when $\Gamma$ and $A$ are clear. 
For a presheaf family, we thus get several notions of fibrancy depending on where we put the limit between the degenerate and the regular part.

- If all the dependency is put in the regular part, we recover the CCHM notion of fibrancy. It corresponds to the fact that, in the empty context, the presheaf $\emptyset \vdash \mathbf{R F i b}(z: \Sigma \Gamma A$. $P)$ has a section. We will say that $P$ is fully fibrant.

- If all the dependency is put in the degenerate part, we get something weaker which corresponds to the fact that $\Gamma, A \vdash$ Fib $P$ has a section, or equivalently that $\Gamma \vdash \operatorname{DFib}(x: A . P)$ has a section. We will say that $P$ is degenerately fibrant.

In the transport rule:

$$
\frac{\Gamma, x: A \vdash P \quad \Gamma \vdash p: t \sim_{A} t^{\prime} \quad \Gamma \vdash H: \mathbf{R F i b}(x . P) \quad \Gamma \vdash u: P t}{\Gamma \vdash \operatorname{transport}(P, H, p, u): P t^{\prime}}
$$

the fibrancy hypothesis is minimal in the sense that only what is needed to interpret the transport is required to be in the regular part.

The notion of regular fibrancy has been generalized to damped horn inclusions by Nuyts (2018). It is called contextual fibrancy there. In particular, Nuyts has shown that there is always a fibrant replacement for contextual fibrancy. We will demonstrate this for our particular case in the coming section.

\section{Fibrant Replacement [FibRepl.v]}

The fibrant replacement is an operator turning an arbitrary type $A$ into a fibrant type $\bar{A}$ satisfying an universal property. Several authors have remarked that the fibrant replacement is not admissible in two-level type theories. For instance, it is inconsistent with the existence of types which are not h-sets (Capriotti 2017) or with an interval (Homotopy Type Theory wiki 2014). Things go nicer in our setting because we use degenerate fibrancy: given a type family $x: A$. $P$, we will only get a family $x: A . \bar{P}$ which is degenerately fibrant, $\mathbf{D F i b}(x, \bar{P})$, and not regularly fibrant, $\mathbf{R F i b}(x . \bar{P})$ (and even less fully fibrant). It turns out that such a degenerate fibrant replacement is admissible. In fact, it can be defined in DTT using a QIT, which is quite remarkable. See Appendix B for the proof that a regular fibrant replacement would be inconsistent.

The fibrant replacement of a type $A$ is the (strict) QIT defined as follows:

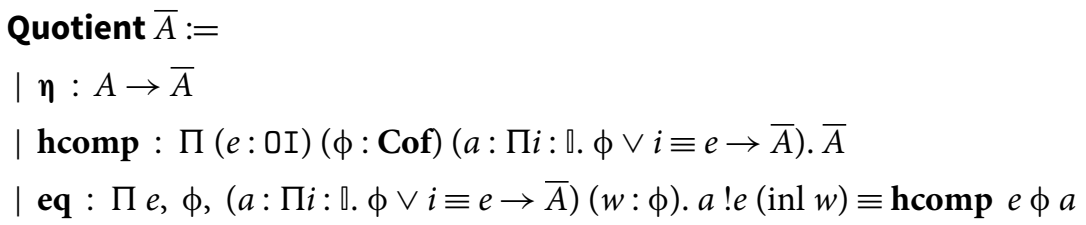

$\eta$ is the embedding of $A$ into $\bar{A}$, hcomp freely adds the compositions needed so that $\bar{A}$ is (degenerately) fibrant, and eq assert than hcomp $e \phi a$ extends $(\phi, a ! e \circ$ inl).

For any type $A, \bar{A}$ is fibrant, but for a type family $x: A . P, x: A . \bar{P}$ is not regularly fibrant in general.

This QIT enjoys the following elimination principle:

$$
z: \bar{A} \vdash P \quad \vdash \eta^{\prime}: \Pi a: A . P\{z:=\eta a\}
$$

$\vdash$ hcomp' $: \Pi e, \phi,(a: \Pi i:$ व. $\phi \vee i \equiv e \rightarrow \bar{A})\left(a^{\prime}: \Pi i, w . P\{z:=a i w\}\right) . P\{z:=\operatorname{hcomp} e \phi a\}$ $\vdash \mathrm{eq}^{\prime}: \Pi e, \phi, a, a^{\prime}, w$. eq $e \phi a w \#^{P} a^{\prime} ! e(\operatorname{inl} w) \equiv \operatorname{hcomp}^{\prime}$ e $\phi a a^{\prime}$

$$
\vdash \text { repl_ind }\left(P, \eta^{\prime}, \text { hcomp }^{\prime}, \mathrm{eq}^{\prime}\right): \Pi z: \bar{A} . P
$$




$$
\begin{aligned}
& \text { repl_ind }\left(P, \eta^{\prime} \text {, hcomp } p^{\prime}, \mathrm{eq}^{\prime}\right)(\eta a) \simeq_{\beta \eta} \eta^{\prime} a \\
& \text { repl_ind } \left.\left(P, \eta^{\prime}, \text { hcomp }^{\prime}, \text { eq }{ }^{\prime}\right) \text { (hcomp e } \phi a\right) \\
& \simeq_{\beta \eta} \operatorname{hcomp}^{\prime} \text { e } \phi a\left(\lambda i, w \text {.repl_ind }\left(P, \eta^{\prime}, \operatorname{hcomp}^{\prime}, \mathrm{eq}^{\prime}\right)(\text { a } i w)\right)
\end{aligned}
$$

From this "raw elimination principle" generated by the quotient, we can prove that the fibrant replacement has another elimination principle, which is the one expected for a fibrant replacement.

Proposition 10. The degenerate fibrant replacement satisfies the following elimination principle:

$$
\begin{array}{cl}
z: \bar{A} \vdash P \quad & \vdash \eta^{\prime}: \Pi a: A . P\{z:=\eta a\} \quad \vdash H: \operatorname{RFib}(z . P) \\
& \vdash \text { repl_ind' }\left(P, \eta^{\prime}, H\right): \Pi z: \bar{A} \cdot P z \\
& \text { repl_ind' }\left(P, \eta^{\prime}, H\right)(\eta a) \simeq_{\beta \eta} \eta^{\prime} a
\end{array}
$$

Proof. repl_ind' $\left(P, \eta^{\prime}, H\right)$ is defined by induction on the quotient (using repl_ind). In the case of hcomp $e \phi a$, modulo strict rewritings, the provided term is given by a composition in $P$ :

$$
\operatorname{comp}_{P} \text { e } a_{0} \phi p_{0}
$$

with $a_{0}:=\lambda i$. hcomp $e(\phi \vee(i \equiv e))\left(\lambda j w . a\left(i \sqcap_{e} j\right)(\ldots \# w)\right)$

and $p_{0}:=\lambda i w \ldots \# w$

where $\Pi_{0}:=\sqcap$ and $\Pi_{I}:=\sqcup$.

Instantiated with a constant type family _ $: A$. $B$, we get the non-dependent elimination principle asserting that for every map $f: A \rightarrow B$, if $B$ is fibrant, then $f$ can be factorized as:

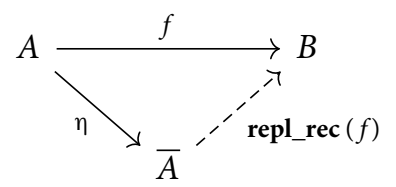

and the triangle commutes definitionally.

With this non-dependent elimination principle we can lift every map $f: A \rightarrow B$ to a map $\bar{f}$ : $\bar{A} \rightarrow \bar{B}$ and we have the following equalities:

$$
\bar{f}(\eta x) \simeq_{\beta \eta} \eta(f x) \quad \overline{g \circ f} \equiv \bar{g} \circ \bar{f} \quad \overline{\mathbf{i d}_{A}} \equiv \mathbf{i d} \bar{A}_{\bar{A}}
$$

The two strict equalities are proven by induction on the quotient, using the first elimination principle repl_ind.

Last, we will need the property that any type family $P: A \rightarrow \mathscr{U}$ regularly fibrant can be extended to $\bar{A}$ preserving regular fibrancy. We call the property the extension rule. Unfortunately, we only know how to prove it in the empty context (the proof relies on the universe of fibrant types). We cannot be certain that it holds in an arbitrary context, but we were not able to derive an inconsistency neither. In the remaining of this paper, we will take care to use it only in an empty context. The propositions only valid in an empty context will be marked by an empty set context symbol "valid in $\emptyset \mathrm{ctx}$ ".

Proposition 11 (extension rule, valid in $\emptyset \mathbf{c t x}$ ). For every type family $\emptyset \vdash P: A \rightarrow \mathscr{U}$ which is regularly fibrant (RFib $P$ ), there exists a type family $\emptyset \vdash \widetilde{P}: \bar{A} \rightarrow \mathscr{U}$ such that:

$$
\widetilde{P} \circ \eta \equiv P \quad \text { and } \quad \text { RFib } \widetilde{P}
$$

The proof relies on the existence of a universe of fibrant types $\mathscr{U} \mathscr{F}$ constructed in Cohen et al. (2017) and the fact that it is fibrant. This universe classifies fully fibrant type families. $\mathscr{U} \mathscr{F}$ has not to be confused with $\mathscr{U} \mathscr{H}$ defined as $\Sigma A: \mathscr{U}$. Fib $A$ and which classifies only degenerately 
fibrant families. The interest of $\mathscr{U} \mathscr{F}$ lies in the fact that it is univalent (which is not the case for $\mathscr{U} \mathscr{H}$ : it even has an extension structure). Note that $\mathscr{U} \mathscr{F}$ is not definable internally in $\square \mathrm{TT}$, doing so requires to move to a more complicated setting, Crisp type theory, which we do not detail here. See Licata et al. (2018) for more on this topic.

Proof. Let $P: A \rightarrow \mathscr{U}$ be a regularly fibrant family in the empty context. Since the context is empty, $P$ is in fact fully fibrant. This fibrancy structure give a map:

$$
P^{\prime}: A \rightarrow \mathscr{U} \mathscr{F}
$$

such that $\mathrm{El} \circ P^{\prime} \equiv P$. But the universe $\mathscr{U} \mathscr{F}$ is itself fibrant, hence we have the map:

$$
\text { repl_rec }\left(P^{\prime}\right): \bar{A} \rightarrow \mathscr{U} \mathscr{F}
$$

We take $\widetilde{P}$ to be El $\circ$ repl_rec $\left(P^{\prime}\right)$. By the fibrancy rule of El, this type family is fully fibrant, and hence regularly fibrant.

The extension rule has a very strong consequence, it allows transporting along an equality $\eta x=$ $\eta y$ to get a map $P x \rightarrow P y$ if $P$ is regularly fibrant.

Proposition 12. The following operator $\overline{J_{=}}$is derivable:

$$
\begin{gathered}
\vdash P: \Pi x: A \cdot \eta t={ }_{A} \eta x \rightarrow \mathscr{U} \quad \vdash H: \mathbf{R F i b}_{2}(x, p . P) \\
\vdash p: \eta t={ }_{A} \eta t^{\prime} \quad \vdash u: P t\left(\operatorname{refl}_{=} \eta t\right)
\end{gathered} \quad \overline{J_{=}}\left(P, H, \mathbf{r e f l}_{=} t, u\right) \equiv u
$$

Proof. $\overline{J_{=}}$is obtained by applying $J_{=}$to $\widetilde{P}$.

Let us finish this section by remarking that the extension rule is a consequence of Sattler's fibration extension property (Sattler 2017, Corollary 7.7). Indeed, this one gives that for any type family $x: A$. $P$ and acyclic cofibration $f: B \rightarrow A$ (still in the empty context), if $P \circ f$ is regularly fibrant, then so is $P$.

\section{Model Category}

The fibrant replacement is one of the major ingredients to define a pre-model structure on the universe $\mathscr{U}$. This will be done in the next sections, but first, we recall what is a model category. We use here a type theoretic setting, see Hirschhorn (2009), Hovey (2007) which are standard references for a set theoretic account.

Defining the right notion of category in HoTT with a relevant equality is quite intricate as several choices can be made to tame higher coherences. There is no such shilly-shallying when a strict equality is used, as already noticed in Altenkirch et al. (2016). In fact, everything takes place exactly as in set theory.

Definition 4. A category consists of:

- a type $\mathscr{C}$ of objects,

- for all $I, J: \mathscr{C}$, a type $\operatorname{Hom}(I, J)$ of arrows

- for all $I: \mathscr{C}$, an identity arrow $\mathbf{i d}_{A}: \operatorname{Hom}(I, I)$

- for all $I, J, K: \mathscr{C}$, a composition function:

${ }_{-}{ }_{-}: \operatorname{Hom}(J, K) \rightarrow \operatorname{Hom}(I, J) \rightarrow \operatorname{Hom}(I, K)$

- for all $f: \operatorname{Hom}(I, J)$, a proof off $\circ \circ \mathbf{i d}_{I} \equiv f$ and $\mathbf{i d}_{J} \circ f \equiv f$

- for all $f: \operatorname{Hom}(I, J), g: \operatorname{Hom}(J, K), h: \operatorname{Hom}(K, L)$, a proof of $h \circ(g \circ f) \equiv(h \circ g) \circ f$

We write $\mathscr{C}$ both for the category and the type of its objects, and $g f$ for the composition of arrows $g \circ f$. 
With this definition, each universe $\mathscr{U}$ forms a category where $\operatorname{Hom}(A, B)$ is given by $A \rightarrow B$. Identity and composition are those of functions and the laws are given by $\beta \eta$ conversion. We write Type this category. In the following, we fix $\mathscr{C}$ a category.

Definition 5. Let $f: \operatorname{Hom}(X, Y)$ and $g: \operatorname{Hom}\left(X^{\prime}, Y^{\prime}\right)$ be arrows of $\mathscr{C}$. We say that $f$ is a retract of $g$ if there exists arrows $s, r, s^{\prime}$, and $r^{\prime}$ such that the following diagram commutes

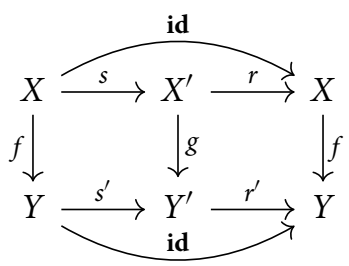

Here and in the rest of the paper, "exists" is understood in the constructive sense, that is, as a sigma type (witnessed existence) and not as a squashed sigma type (mere existence).

By a class of arrows of $\mathscr{C}$, we simply mean a type family $X, Y: \mathscr{C}, f: \operatorname{Hom}(X, Y)$. P over arrows of $\mathscr{C}$. We write $f \in P$ for all function $f$ such that $P$ is inhabited. If $Q$ is another class, we write $P \leftrightarrow Q$ if we have $\Pi X, Y$. $\Pi f: \operatorname{Hom}(X, Y) . P f \leftrightarrow Q f$; and $P \cap Q$ for the conjunction of the two classes.

Definition 6. A class $P$ of arrows of $\mathscr{C}$ satisfies the 2-out-of-3 property if, for all arrows $X \stackrel{f}{\longrightarrow} Y \stackrel{g}{\longrightarrow} Z$ such that if two of $f, g$, and $g \circ f$ belong to $P$, so does the third. More precisely, it means that we have three functions:

- $\Pi f, g . P f \rightarrow P g \rightarrow P(g \circ f)$

- $\Pi f, g . P(g \circ f) \rightarrow P f \rightarrow P g$

- $\Pi f, g . P g \rightarrow P(g \circ f) \rightarrow P f$

Definition 7. Let $f: \operatorname{Hom}(X, Y)$ and $g: \operatorname{Hom}\left(X^{\prime}, Y^{\prime}\right)$ be arrows of $\mathscr{C}$. It is said that $f$ has the left lifting property $(L L P)$ with respect to $g$ (and that $g$ has the right lifting property $(R L P)$ with respect to $f$ ) if, for all arrows $F: \operatorname{Hom}\left(X, X^{\prime}\right)$ and $G: \operatorname{Hom}\left(Y, Y^{\prime}\right)$ such that the square below commutes, there exists an arrow $\gamma: \operatorname{Hom}\left(Y, X^{\prime}\right)$ making the two triangles commute

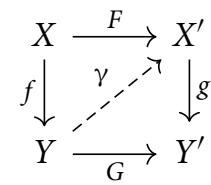

We then say that an arrow $f$ has the LLP (resp. the RLP) with respect to a class of arrows $P$ if it has it with respect to all arrows of $P$. We write $\mathbf{L L P}(P)(\operatorname{resp} \operatorname{RLP}(P))$ the class of such arrows.

Definition 8. A weak factorization system on $\mathscr{C}$ consists of two classes of arrows $L$ and $R$ such that:

- every arrow $f$ of $\mathscr{C}$ can be factorized as $f \equiv r \circ l$ with $l \in L$ and $r \in R$

- $L$ is exactly the class of arrows of $\mathscr{C}$ which have the LLP with respect to $R$ :

$$
L \leftrightarrow \mathbf{L L P}(R)
$$

- $R$ is exactly the class of arrows of $\mathscr{C}$ which have the $R L P$ with respect to $L$ :

$$
R \leftrightarrow \mathbf{R L P}(L)
$$

The classes $L$ and $R$ of a weak factorization system enjoy several good properties: they contain all isomorphisms, they are closed under retract, $L$ is closed under pushouts, $R$ is closed under pullbacks, etc. 
We can now define what is model category.

Definition 9. A pre-model structure on $\mathscr{C}$ consists of three classes of arrows $F, C$, and $W-$ the fibrations, the cofibrations, and the weak equivalences - such that:

- W satisfies the 2-out-of-3 property

- $(A C, F)$ and $(C, A F)$ are two weak factorization systems, where $A C:=C \cap W$ and $A F:=F \cap W$.

The arrows of $A C$ (resp. AF) are called the acyclic cofibrations (resp. acyclic fibrations).

Definition 10. A model category is a category equipped with a pre-model structure which is complete (it has all small limits) and cocomplete (it has all small colimits).

The rest of this article is devoted to showing that there is a pre-model structure on the category Type.

\section{7. (AC,F) Weak Factorization System}

The fibrant replacement allows us to define the first factorization system, given by homotopy fibers, and weak equivalences. Our design choices are guided by the following definition of fibrations.

Definition 11. A function $f: A \rightarrow B$ is said to be a fibration if there exists a regularly fibrant type family $x: X . P$ such that $f$ is a retract of $\pi_{1}:(\Sigma x: X . P) \rightarrow X:$

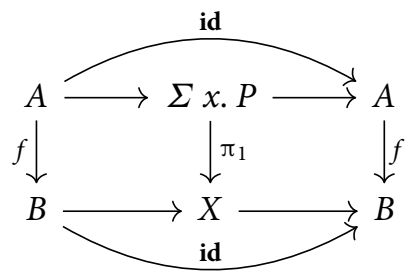

We write $F$ the class of fibrations. The class of acyclic cofibrations is defined to be $\mathbf{L L P}(F)$ for the moment, we will check that it coincides with $C \cap W$ afterward.

Let us recall that for a map $f: A \rightarrow B$, its homotopy fiber in $y: B$ is the type defined by:

$$
\text { fiber }_{f} y:=\Sigma x: A \cdot f x={ }_{B} y
$$

Then, every function $f: A \rightarrow B$ factorizes through the fibers of its fibrant replacement:

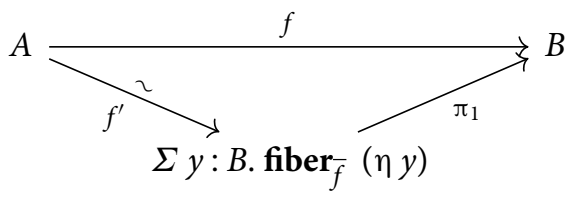

with $f^{\prime}:=\lambda x: A .\left(f x, \eta x, \operatorname{refl}_{=}(\eta(f x))\right)$. We add the fibrant replacement so that the type family fiber $_{f}$ is regularly fibrant (and thus fiber $_{f} \circ \eta$ also).

Proposition 13 (valid in $\emptyset \mathbf{c t x}) .(\operatorname{LLP}(F), F)$ is a weak factorization system on $\mathscr{U}$. 
Proof. We have to check that:

- for all $f, \pi \in F$ and $f^{\prime} \in \operatorname{LLP}(F)$

- $\mathbf{L L P}(F) \leftrightarrow \operatorname{LLP}(F)$

- $F \leftrightarrow \operatorname{RLP}(\operatorname{LLP}(F))$

The only two nontrivial points are that:

- for all $f, f^{\prime} \in \mathbf{L L P}(F)$

- $\mathbf{R L P}(\mathbf{L L P}(F)) \subseteq F$

We only give the proof of the first one. Many proofs of the following sections are similar, and they all can be found in the formalization.

As the lifting property is stable under retracts, to show that $f^{\prime} \in \mathbf{L L P}(F)$ we only have to solve the following lifting problem:

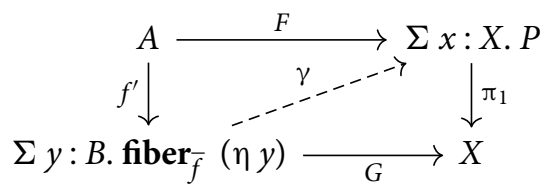

with $x . P$ a regularly fibrant family.

The map $\gamma$ is defined as the composition:

$$
\Sigma y: B . \operatorname{fiber}_{\bar{f}}(\eta y) \stackrel{(\mathrm{id}, \alpha)}{\longrightarrow} \Sigma w . P\{x:=G w\} \stackrel{\beta}{\longrightarrow} \Sigma x: X . P
$$

where $\beta(w, z):=(G w, z)$. To provide $\alpha$, we need a map of type:

$$
\Pi(y: B)\left(x^{\prime}: \bar{A}\right)\left(p: \bar{f} x^{\prime}=\eta y\right) . P\left\{x:=G\left(y, x^{\prime}, p\right)\right\}
$$

Using the fibrancy rules of $\Pi$ and $=$, we can now do an induction on the fibrant replacement ( $P$ is regularly fibrant and $\bar{B}$ if fibrant). Hence, we need

$$
\Pi(y: B)(x: A)(p: \eta(f x)=\eta y) . P\{x:=G(y, \eta x, p)\}
$$

Then, using the path induction principle given by the extension rule (Proposition 12), it becomes

$$
\Pi x: A . P\left\{x:=G\left(f x, \eta x, \operatorname{refl}_{=}\right)\right\}
$$

which is given by $F$. We see here that the extension rule is crucial to have the lifting. Remark also that identity types are needed to have the commutation of the left triangle that would not hold with path types.

\subsection{Weak equivalences [Equivalences.v]}

To define weak equivalences, we use HoTT equivalences. Let us recall that the predicate of being an equivalence $\operatorname{IsEquiv}(f)$ is defined to be:

$$
\Sigma(g: B \rightarrow A)(\eta: \Pi x . g(f x)=x)(\varepsilon: \Pi x . f(g x)=x) . \Pi x . \mathbf{a p}_{f}(\eta x)=\epsilon(g x)
$$

In Orton and Pitts (2018a), Orton and Pitts use path types to define equivalences. Here, we choose to use identity types because it implies less conversions between paths and identity types in the following. The two types are logically equivalent but we do not know which one is more meaningful. 
Definition 12. A map $f: A \rightarrow B$ is a weak equivalence if $\bar{f}: \bar{A} \rightarrow \bar{B}$ is an equivalence:

$$
W(f):=\operatorname{IsEquiv} \bar{f}
$$

Here, the fibrant replacement seems to play a role similar to geometric realization. In homotopy theory, the usual model structure on simplicial sets has, for weak equivalences, the maps whose geometric realization in Top are weak equivalences.

\section{Pushouts and Cylinders [Cylinder.v]}

For the second factorization system, we need cylinders, which are the dual of fibers. We define cylinders from homotopy pushouts, so let us first define homotopy pushouts.

\subsection{Pushout}

In HoTT, homotopy pushouts are an instance of HITs. Recently, Coquand et al. gave the interpretation of several HIT, including pushouts, in the CCHM model (Coquand et al. 2018). We replay here their construction in light of the fibrant replacement.

Let us fix the following span of which we want to construct the pushout:

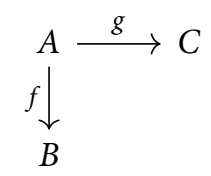

The pushout of $f$ and $g$ is the following HIT:

$$
\begin{aligned}
& \text { HIT } B \sqcup_{A} C:= \\
& \text { | pol : } B \rightarrow B \sqcup_{A} C \\
& \text { | por : } C \rightarrow B \sqcup_{A} C \\
& \text { | poq : } \Pi x: A \cdot \operatorname{pol}(f x)={ }_{B \sqcup_{A} C} \operatorname{por}(g x)
\end{aligned}
$$

Remark. The homotopy pushout should not be confused with the strict pushout given by a strict quotient.

Now we want to define this HIT in QTT. The construction goes in two steps: first, we define a "naive" strict quotient and then we take the fibrant replacement. Let $\mathbf{P O}_{\mathbf{0}}$ be the following strict quotient:

$$
\begin{aligned}
& \text { Quotient } \mathrm{PO}_{0}:= \\
& \text { | } \mathbf{p o l}_{\mathbf{0}}: B \rightarrow \mathbf{P O}_{\mathbf{0}} \\
& \text { | } \text { por }_{\mathbf{0}}: C \rightarrow \mathbf{P O}_{\mathbf{0}} \\
& \text { | } \operatorname{poq}_{0}: A \rightarrow \square \rightarrow \mathbf{P O}_{\mathbf{0}} \\
& \text { | } \operatorname{poq}_{1}: \Pi x: A \cdot \operatorname{poq}_{\mathbf{0}} x 0 \equiv \mathbf{P O}_{\mathbf{0}} \operatorname{pol}_{\mathbf{0}}(f x) \\
& \text { | } \operatorname{poq}_{\mathbf{r}}: \Pi x: A \cdot \operatorname{poq}_{\mathbf{0}} x 1 \equiv \mathbf{P O}_{0} \operatorname{por}_{\mathbf{0}}(g x)
\end{aligned}
$$

pol $_{\mathbf{0}}$ and por $_{\mathbf{0}}$ interpret pol and por, and $\operatorname{poq}_{\mathbf{0}} x$ is a path from $\operatorname{pol}_{\mathbf{0}}(f x)$ to $\operatorname{por}_{\mathbf{0}}(g x)$. The endpoints agree with the strict equalities by which we quotient. We will write $\mathbf{P O}_{\mathbf{0} \_ \text {ind }}$ the eliminator generated by the strict quotient. 
Unfortunately, this type is never fibrant and hence we take the fibrant replacement:

$$
\begin{aligned}
B \sqcup_{A} C & :=\overline{\mathbf{P O}_{\mathbf{0}}} \\
\text { pol }: B \rightarrow B \sqcup_{A} C & :=\eta \circ \mathbf{p o l}_{\mathbf{0}} \\
\text { por }: C \rightarrow B \sqcup_{A} C & :=\eta \circ \operatorname{por}_{\mathbf{0}} \\
\operatorname{poq}: \Pi x: A \cdot \operatorname{pol}(f x) \sim_{B \sqcup_{A} C} \operatorname{por}(g x) & :=\operatorname{ap}_{\eta}\left(\operatorname{poq}_{\mathbf{0}}, \operatorname{poq}_{\mathbf{l}}, \operatorname{poq}_{\mathbf{r}}\right)
\end{aligned}
$$

Let us remark that poq is a path and not an inhabitant of an identity type. Hence, if we want to recover an identity type, we have to apply paths2id. In general, the interaction between HIT and identity types remains to be studied.

As in the case of the fibrant replacement, we can define the expected elimination principle from the raw elimination principle $\mathbf{P O}_{\mathbf{0}}$ ind.

Proposition 14. $B \sqcup_{A} C$ satisfies the following elimination principle:

$$
\begin{gathered}
w: B \sqcup_{A} C \vdash P \quad \vdash H: \operatorname{RFib}(w . P) \\
\vdash \operatorname{pol}^{\prime}: \Pi b: B . P\{w:=\operatorname{pol} b\} \quad \vdash \operatorname{por}^{\prime}: \Pi c: C . P\left\{w:=\operatorname{por}^{\prime} c\right\} \\
\vdash \operatorname{poq}^{\prime}: \Pi x: A . \operatorname{transport}\left(P, \operatorname{poq} x, \operatorname{pol}^{\prime}(f x)\right) \sim_{P(\operatorname{por}(g x))} \operatorname{por}^{\prime}(g x) \\
\hline \vdash \text { PO_ind }\left(P, H, \operatorname{pol}^{\prime}, \operatorname{por}^{\prime}, \operatorname{poq}^{\prime}\right): \Pi w: B \sqcup_{A} C . P w \\
\text { PO_ind }\left(P, H, \operatorname{pol}^{\prime}, \operatorname{por}^{\prime}, \operatorname{poq}^{\prime}\right)(\operatorname{pol} b) \simeq_{\beta \eta} \operatorname{pol}^{\prime} b \\
\text { PO_ind }\left(P, H, \operatorname{pol}^{\prime}, \operatorname{por}^{\prime}, \operatorname{poq}^{\prime}\right)(\operatorname{por} c) \simeq_{\beta \eta} \operatorname{por}^{\prime} c
\end{gathered}
$$

Note that the transport in poq' is a transport along a path.

Proof. The proof goes by induction on the fibrant replacement using repl_ind' $(P$ is regularly

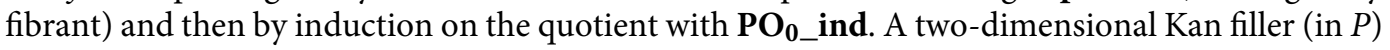
is needed to complete the case of the $\mathbf{p o q}_{\mathbf{0}}$. See the formalization.

Remark. For the non-dependent eliminator, we have a strict computational rule on poq:

$$
{\text { appo_rec }\left(P, H, \text { pol'}^{\prime}, \text { por' }^{\prime}, \operatorname{poq}^{\prime}\right)}(\mathbf{p o q} x) \equiv \operatorname{poq}^{\prime} x
$$

Even if we expect it, we do not know, at the time of writing, if a similar strict equality holds for the dependent eliminator.

Pushout satisfies the following fibrancy rule.

Proposition 15. If $x . A, x . B, x . C$ are regularly fibrant type families over $X$. Then for all maps $f: \Pi x: X . A \rightarrow B$ and $g: \Pi x: X . A \rightarrow C$, the type family given by the pushout $x: X . B \sqcup_{A} C$ is regularly fibrant:

$$
\frac{\vdash \mathbf{R F i b}(x . A) \quad \vdash \mathbf{R F i b}(x . B) \quad \vdash \mathbf{R F i b}(x . C)}{\vdash \operatorname{RFib}\left(x . B \sqcup_{A} C\right)}
$$

This property is quite remarkable because, in the general case, the pointwise fibrant replacement $x . \bar{A}$ has no hope to be regularly fibrant. However, it is the case for this particular family $x . \overline{\mathbf{P O}_{\mathbf{0}}}$. The secret lies in a decomposition of regular fibrancy into degenerate fibrancy and transport structure. This decomposition was discovered by Coquand et al. (2018). Cavallo and Harper (2019) also use a similar decomposition to give a semantic to wide class of HITs in computational cubical theory. 
Definition 13. A transport structure on a type family $x: A$. P is an inhabitant of the following type:

$$
\begin{aligned}
\operatorname{Trans}(x . P):=\Pi & (e: \mathrm{OI})(a: \rrbracket \rightarrow A)(\phi: \text { Cof })(\operatorname{cst}: \phi \rightarrow \Pi i . \llbracket a e \equiv a i) \\
& \left(p_{0}: P\{x:=a e\}\right) . \Sigma p_{1}: P\{x:=a ! e\} . \Pi w . \phi \operatorname{cst} w ! e \# p_{0} \equiv p_{1}
\end{aligned}
$$

It means that there is a composition operation along all paths a which are constant on the face $\phi$ considered.

Proposition 16. A type family x. $P$ is regularly fibrant if and only if it is degenerately fibrant and has a transport structure:

$$
\operatorname{RFib}(x . P) \leftrightarrow \operatorname{DFib}(x . P) \times \operatorname{Trans}(x . P)
$$

Proof. See Coquand et al. (2018).

Proposition 17. The fibrant replacement preserves the transport structure:

$$
\operatorname{Trans}(x . P) \rightarrow \operatorname{Trans}(x . \bar{P})
$$

Proof. The following argument comes from Cavallo and Orton. ${ }^{5}$

This property is true for any endofunctor ${ }^{6}$ on types, that is to say, for any operator $R$ on types such that there is an operator:

$$
F_{A, B}:(A \rightarrow B) \rightarrow(R A \rightarrow R B)
$$

with $F \operatorname{id}_{A} \equiv \operatorname{id}_{R A}$ and $F(g \circ f) \equiv F g \circ F f$. We have seen that such equalities hold for the fibrant replacement.

The transport operation on $P$ gives a map $P\{x:=a e\} \rightarrow P\{x:=a ! e\}$. By applying $F$, we get a map $R(P\{x:=a e\}) \rightarrow R(P\{x:=a e\})$. The fact that $F$ preserves identity maps is enough to show that it actually gives a transport structure on $x . R P$.

The pushout is degenerately fibrant (because of the fibrant replacement), hence it remains to show that it has a transport structure.

Proposition 18. $x . \overline{\mathbf{P O}_{0}}$ has a transport structure.

Proof. See Coquand et al. (2018) or the formalization in Cylinder.v.

\subsection{Cone}

For any type $A$, the cone of $A$ is the following homotopy pushout:

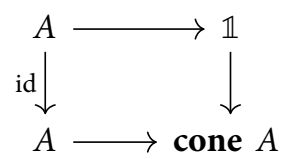

So we define cone $A:=A \sqcup_{A} \mathbb{1}$. It can also be seen directly as an HIT:

$$
\begin{aligned}
& \text { HIT cone } A:= \\
& \mid \text { inc }: A \rightarrow \text { cone } A \\
& \mid \text { point }: \text { cone } A \\
& \mid \text { eq }: \Pi x: A \text {. inc } x=\text { cone } A \text { point }
\end{aligned}
$$

The cone satisfies the following elimination principle:

$$
\begin{gathered}
\begin{array}{c}
w: \text { cone } A \vdash P \quad \vdash H: \mathbf{R F i b}(w . P) \quad \vdash \text { inc }^{\prime}: \Pi x: A . P\{w:=\text { inc } x\} \\
\vdash \text { point }
\end{array}: P\{w:=\text { point }\} \quad \vdash \mathrm{eq}^{\prime}: \Pi x: A . \operatorname{transport}\left(P, \text { eq } x, \text { inc }^{\prime} x\right) \sim \text { point }^{\prime} \\
\vdash \text { cone_ind }\left(P, H, \text { inc }^{\prime}, \text { point }^{\prime}, \mathrm{eq}^{\prime}\right): \Pi w: \text { cone } A . P w
\end{gathered}
$$


The cone enjoys the expected fibrancy rule $\operatorname{RFib}(x . A) \rightarrow \operatorname{RFib}(x$. cone $(A x))$.

Proposition 19. A cone is always contractible. For any type A we have

\section{Contr(cone A)}

Proof. By induction with cone_ind.

\subsection{Cylinder}

At last, we define the mapping cylinder. Let $f: A \rightarrow B$ be a map and $y: B$. The mapping cylinder of $f$ in $y$ is

$$
\operatorname{Cyl}_{f} y:=\operatorname{cone}(\Sigma x: A . f x=y)
$$

Then, the cylinder satisfies the following introduction and elimination rules:

$$
\begin{aligned}
& \text { top : } \Pi x: A . \mathbf{C y l}_{f}(f x) \\
& :=\lambda x: A \cdot \operatorname{inc}\left(x, \operatorname{refl}_{=}(f x)\right) \\
& \text { base : } \Pi y: B . \mathbf{C y l}_{f} y \\
& :=\lambda y: B \text {. point } \\
& \text { cyleq : } \Pi x: A \text {. top } x \sim_{\mathrm{Cyl}(f x)} \text { base }(f x) \\
& :=\lambda x: A . \mathbf{e q}\left(x, \operatorname{refl}_{=}(f x)\right) \\
& y: B, w: \mathbf{C y l}_{f} y \vdash P \quad \vdash H: \mathbf{R F i b}_{\mathbf{2}}(y, w . P) \\
& \vdash \text { top }^{\prime}: \Pi x: A . P\{y:=f x, w:=\text { top } x\} \quad \vdash \text { base }^{\prime}: \Pi y: B . P\{w:=\text { base } y\} \\
& \vdash \text { cyleq }: \Pi x: A \text {. transport }(P, \text { cyleq } x \text {, top } x) \sim \operatorname{base}^{\prime}(f x) \\
& \vdash \text { cyl_ind }\left(P, H \text {, top }{ }^{\prime}, \text { base }^{\prime}, \text { cyleq }^{\prime}\right): \Pi(y: B)\left(w: \mathbf{C y l}_{f} y\right) . P
\end{aligned}
$$

Proposition 20. Let $A$ and $B$ be regularly fibrant families over $X$. Then, Cyl is regularly fibrant as a three variables type family:

$$
\mathbf{R F i b}_{3}\left(x: X, f: A x \rightarrow B x, y: B x . \mathbf{C y l}_{f} y\right)
$$

Last, as a cylinder is cone, all cylinders $\mathbf{C y l}_{f} y$ are contractible.

\section{9. (C,AF) Weak Factorization System [Model_structure.v]}

We can now describe the second factorization system. Every function $f$ factorizes as:

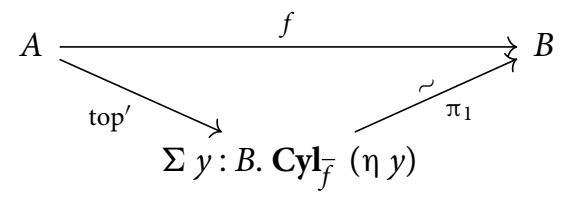

where top ${ }^{\prime}:=\lambda x .\left(f x, \operatorname{top}_{\bar{f}}(\eta x)\right)$.

With respect to the factorization that Lumsdaine (2011) gave for the fibrant fragment, the fibrant replacement is added. It is added so that the family $y$. $\mathbf{C y l}_{f}(\eta y)$ is regularly fibrant.

The need for the contractibility of cylinders comes from the following characterization of acyclic fibrations.

Proposition 21 (valid in $\emptyset \mathbf{c t x}$ ). A map $f: A \rightarrow B$ is an acyclic fibration (i.e. both a fibration and a weak equivalence) if and only if there exists a regularly fibrant type family $x: X$. $P$ with contractible fibers $(\Pi x: X$. Contr $P)$ such that $f$ is a retract of $\pi_{1}:(\Sigma x: X . P) \rightarrow X$ : 


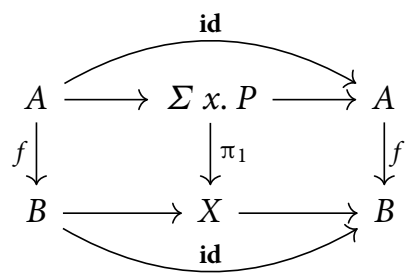

We write AF the class of acyclic fibrations. The class of cofibrations is defined as $\operatorname{LLP}(A F)$.

Remark 22. With this definition of cofibrancy, all objects $A$ : $\mathscr{U}$ are cofibrant (in the sense that the map $\perp \rightarrow A$ is a cofibration).

Proposition 23 (valid in $\emptyset \mathbf{c t x}$ ). ( $\operatorname{LLP}(A F), A F)$ is a weak factorization system on $\mathscr{U}$.

\section{1 (C,AF) factorization through partial elements [Other_facto.v]}

In Cohen et al. (2017, Section 9.1), the authors propose another factorization as a cofibration followed by an acyclic fibration. This factorization uses partial elements and does not make use of cylinders.

Let $f: A \rightarrow B$ be a function. Then, it factorizes as:

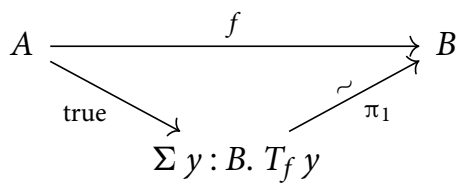

where $T_{f} y:=\Sigma x: \square A . f x \equiv y$ and true $:=\lambda x .\left(f x,\left(\top, \lambda_{-} . x\right), \lambda_{-}\right.$. refl $\left._{\equiv} x\right)$.

Reusing the definitions of classes $F$ (Definition 11) and $\bar{C}$ (Proposition 21), we can rederive the Proposition 23.

Proposition $23^{\prime}$. ( $\left.\operatorname{LLP}(A F), A F\right)$ is a weak factorization system on $\mathscr{U}$.

Proof. We can check that:

(1) $y . T_{f} y$ is always a regularly fibrant family (even if $A$ and $B$ are not fibrant)

(2). for all $y: B, T_{f} y$ is contractible

(3) . true is a cofibration (i.e. lifts against all acyclic fibrations)

The point 2 is an adaptation of the fact that $\square A$ is always contractible. The point 3 uses the characterization of acyclic fibrations of Remark 2.

The proof of 1 requires dependent conjunction in Cof. Cofibrancy of dependent conjunction follows from axiom $\mathbf{a x}_{7}$ and proposition extensionality:

$$
\Pi(\phi: \mathbb{P})(\psi: \phi \rightarrow \mathbb{P}) . \operatorname{cof} \phi \rightarrow(\Pi u \cdot \operatorname{cof}(\psi u)) \rightarrow \operatorname{cof}(\exists u: \phi . \psi u)
$$

We let the reader refer to the formalization to have the details of the proofs.

Let us remark that the proof of this proposition does not rely on the extension rule.

\section{The Model Structure [Model_structure.v]}

We check now that we have taken a correct characterization of acyclic fibrations.

Proposition 24 (valid in $\emptyset \mathbf{c t x}$ ). A map $f: A \rightarrow B$ is in $\operatorname{LLP}(F)$ if and only if it is both a weak equivalence and in $\mathbf{L L P}(A F)$. 
As a consequence, if we plug the two weak factorization systems together we have a pre-model structure.

Theorem 25 (valid in $\emptyset \mathbf{c t x}$ ). There is a pre-model structure on $\mathscr{U}$ where:

- the weak equivalences are maps $f$ such that IsEquiv $\bar{f}$

- the fibrations are retracts of $\pi_{1}:(\Sigma x: X . P) \rightarrow X$ with $P$ regularly fibrant

- the cofibrations are maps which have the LLP with respect to acyclic fibrations

- the (AC,F) factorization off is done through the homotopy fibers of $\bar{f}$

- the (C,AF) factorization off is done through the mapping cylinders of $\bar{f}$.

We could also use factorization through partial elements (Section 9.1) for the (C,AF) factorization.

Remark 26 (not formalized). $\Pi$ types, $\Sigma$ types, and strict equality give all small limits and strict quotients give small colimits. Hence, $\mathscr{U}$ is a model category.

\subsection{Externalization}

Through the interpretation in the CCHM model, the pre-model structure (defined at the level of 『TT, as in Definition 9) on $\mathscr{U}$ provides a pre-model structure (defined externally, as in standard homotopy text books) on the category of cubical sets with connections 啚 (or any other presheaf model of 口TT).

Although we did not formally checked out the details, we conjecture that the pre-model structure on $\mathscr{U}$ gives an alternative description of Sattler's model structure on the category $\widehat{\mathbf{U}}$ (Gambino and Sattler 2015; Sattler 2017) in the sense that the classes F, C, and $W$ are the same.

\subsection{Characterization of the classes}

On the way of proving the previous theorem, we highlighted some characterizations of the four classes $F, C, A F$, and $A C$.

The two factorization systems give prototypical examples of maps which are (acyclic) fibrations and (acyclic) cofibrations. We proved that, in fact, all (acyclic) fibrations and (acyclic) cofibrations arise as retracts, in a canonical sense, of such maps.

Proposition 27 (valid in $\emptyset \mathbf{c t x}$ ). Let $A$ and $B$ be arbitrary types and $f: A \rightarrow B$ a map. Then, we have

- $f$ is a fibration if and only if there exists $j: \Sigma y \cdot \operatorname{fiber}_{\bar{f}}(\eta y) \rightarrow A$ such that $f \circ j \equiv \pi_{1}$ and $j \circ f^{\prime} \equiv \mathbf{i d}_{A}$

- $f$ is an acyclic fibration if and only if there exists $j: \Sigma y \cdot \mathbf{C y l}_{\bar{f}}(\eta y) \rightarrow$ A such that $f \circ j \equiv \pi_{1}$ and $j \circ$ top $^{\prime} \equiv \mathbf{i d}_{A}$

- $f$ is a cofibration if and only if there exists $j: B \rightarrow \Sigma y \cdot \mathbf{C y l}_{\bar{f}}(\eta y)$ such that $j \circ f \equiv$ top $^{\prime}$ and $\pi_{1} \circ j \equiv \mathbf{i d}_{B}$

- $f$ is an acyclic cofibration if and only if there exists $j: B \rightarrow \Sigma y \cdot \mathbf{f i b e r}_{\bar{f}}(\eta y)$ such that $j \circ f \equiv f^{\prime}$ and $\pi_{1} \circ j \equiv \mathbf{i d}_{B}$

where $f^{\prime}$ is $\lambda x .\left(f x, \eta x, \mathbf{r e f l}_{=}(\eta(f x))\right)$ and top ${ }^{\prime}$ is $\lambda x .\left(f x, \mathbf{t o p}_{\bar{f}}(\eta x)\right)$. Diagrams are given in Figure 5. 

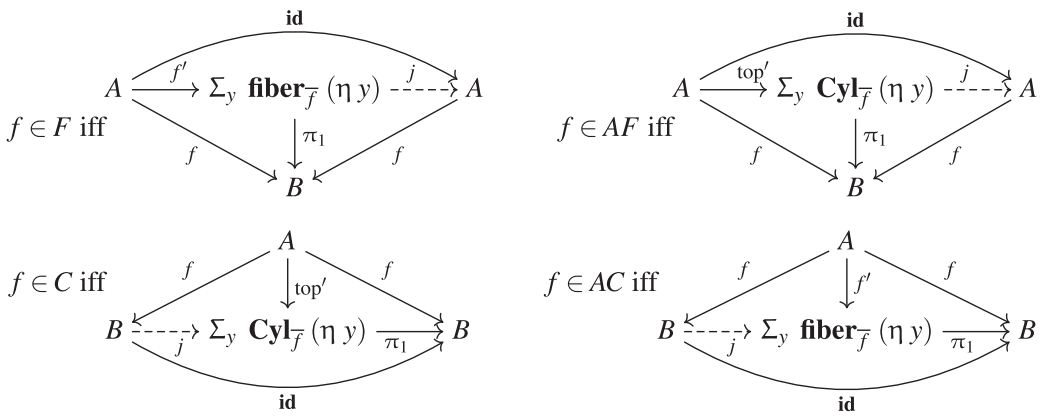

Figure 5. Diagrammatic illustration of Proposition 27.

Proof. The forward implication is given by closure of the four classes under retract. The converse implication is the standard retract argument. ${ }^{7}$

Gambino and Garner (2008) already gave the characterization of fibrations (in the fibrant case) and call such maps type-theoretic normal isofibration.

Acyclic cofibrations have an even better description. As already noticed by Gambino and Garner, they are the injective equivalences. We generalize their definition to encompass nonfibrant types.

Proposition 28. Let $A$ and $B$ be arbitrary types and $f: A \rightarrow B$ a map. Then, $f$ is an acyclic cofibration if and only if it is an injective equivalence, that is, if and only if there exists $r: B \rightarrow \bar{A}$ and

- $\theta: \Pi x: A \cdot r(f x) \equiv \eta_{A} x$

- $\varepsilon: \Pi y: B . \bar{f}(r y)=\eta_{B} y$

- $\alpha: \Pi x:$. strict2id $\left(\mathbf{a p}_{\equiv} \bar{f}(\theta x)\right)=\varepsilon(f x)$.

where strict $2 \mathbf{i d}$ is the map $x \equiv y \rightarrow x=y$.

The last condition means that $\varepsilon(f x)$ is "identity-equal" to $\mathbf{r e f l}_{=}$modulo strict equality rewritings.

\section{Formalization}

We used the Coq proof assistant (Coq Development Team 2018) to simulate UTT. In this way, we have been able to formalize all the results stated in 『TT in this paper except the extension rule.

For this purpose, we have used:

- The Prop universe for $\mathbb{P}$. Proof irrelevance and propositional extensionality are postulated as axioms.

- The equality eq of Coq for the strict equality. It is typed in Prop.

- The usual inductive types of Coq.

- QITs are implemented with a private inductive type and an axiom. This is the (slightly hacky) way HITs are for the moment implemented in Coq.

- The interval and the universe of cofibrant propositions together with the nine axioms are postulated as Coq axioms. See Appendix C.

The paper and the formalization diverge a bit on the universes: there are à la Tarski in the former while Coq's universes are à la Russell. 
The beginning of our formalization is essentially a replay in Coq of Orton and Pitts' Agda formalization (Orton and Pitts 2018b). The formalization in Coq goes pretty well, the only painful thing is to rewrite all strict equalities by hand. The advantage of Coq over Agda is that we can use the rewrite tactic. On the other hand, Orton and Pitts used "rewrite rules" available in Agda to add equalities of the path algebra structure (like " $i \vee 0 \equiv i$ " ...) as definitional equality in Agda. This simplifies the proofs by avoiding some explicit rewritings.

Let us have a quick look at some part of the formalization. The beginning of the definition of the interval is as follows:

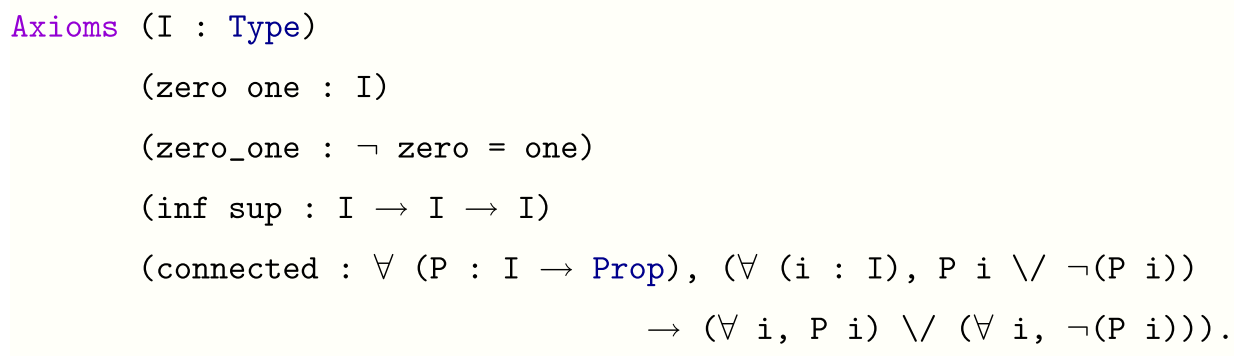

Fibrations are defined by the following record type:

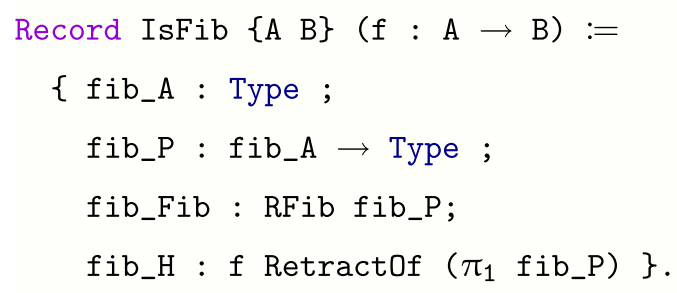

The statement of the main theorem is very short:

Theorem type_model_structure__emptyctx : model_structure TYPE.

And the statements of the characterizations of Section 10.2 look like this:

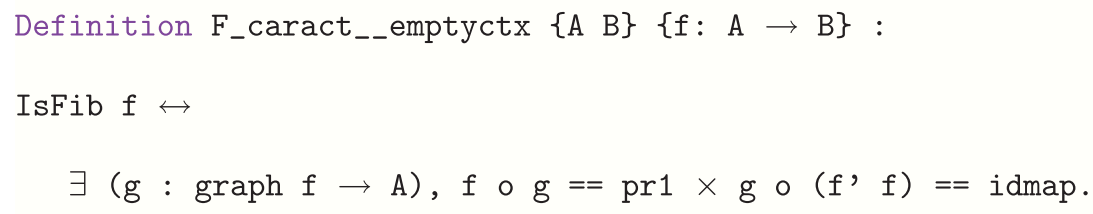

See also Figure 6 for an example of term defined using tactics. 


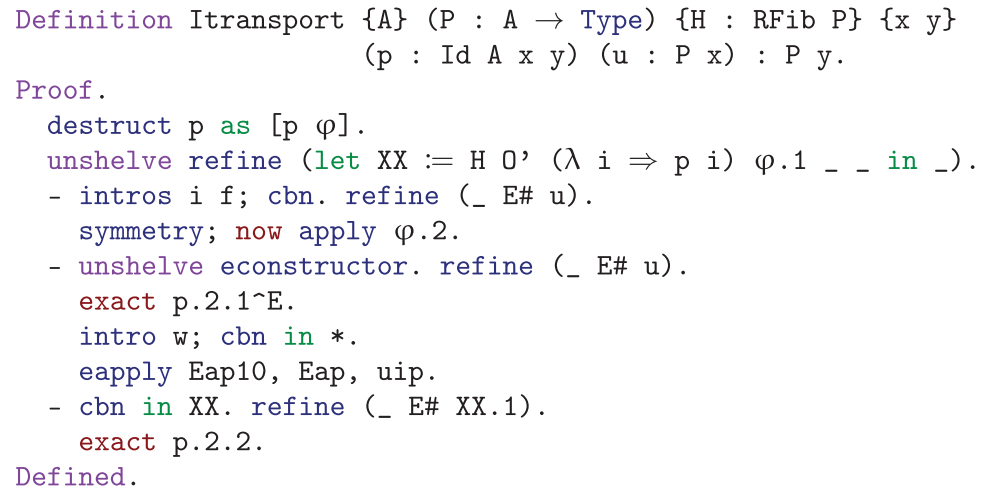

Figure 6. Excerpt of the formalization: transport for identity types.

Remark. We kept track of the uses of the extension rule, which is valid only in the empty context, as carefully as possible. The extension rule is postulated as an axiom and every lemma depending on it has been postfixed with _- emptyctx. A mechanized check would require a proof assistant supporting a modality, such as Agda-Flat (Licata et al. 2018).

The axioms on which the formalization is relying on can be obtained with the Coq command Print Assumptions. This command crawls recursively the environment and looks for the axioms or admitted lemmas on which a definition is relying. Its output for the Theorem 25 and the four characterizations is given in Appendix C. The first group is the set of assumptions on the equality of the meta-theory (Propext, Proof Irrelevance, and Funext). The second group is the set of axioms used to model $\square \mathrm{TT}$. The third group is the set of axioms used to implement strict quotient types (for the fibrant replacement and homotopy pushouts). The last assumption is the extension rule (Proposition 11) and is only valid in the empty context (see the remark above).

\section{Conclusion and Future Work}

『TT, an extension of the internal language developed by Orton and Pitts (2018a), has been used to show internally that there is a pre-model structure on the universe of all types. For this work, we have extended the internal language of Orton and Pitts with a degenerate fibrant replacement, which is definable by a QIT ( for the strict equality). Our results have been formalized in the Coq proof assistant and, as a by product, we provide a formalization of fibrancy of pushouts, as developed by Coquand et al. (2018).

There are several lines for future work. First, the comparison with the pre-model structure (externally) defined by Sattler (2017) remains to be worked out. Our model structure externalizes as a pre-model structure on the category of cubical sets with connections which seems to correspond to Sattler's one, although we have not worked out the details. The extension rule remains to be better understood: is it valid in an non-empty context? Our formalization in Coq does not check that results only valid in the empty context (such as the extension rule) are not used in another context. A possible way to remedy this limitation is to use Andrea Vezzosi's Agda-Flat as in Licata et al. (2018). About weak factorization systems, one may also wonder how the two (C,AF) weak factorization systems that we have presented relate to each other. Then, functoriality or algebraicity of our factorization systems also remains to be investigated. Finally, our work could be generalized to other cube categories using other internal languages, such as in Angiuli et al. (2019). 


\section{Notes}

1 The category of Kan simplicial sets is a model of well-behaved topological spaces.

2 Impredicativity does not seem really necessary in our context (see e.g. Licata et al. 2018) but we choose to stick to Coq metatheory.

3 As opposed to degenerately fibrant, it has nothing to do with regularity in the sense of "transport along reflexivity is definitionally the identity".

4 It comes from degeneracies, see the discussion in Section 4.1.

5 https://github.com/IanOrton/cubical-topos-experiments/blob/master/src/hcomp-trans.agda.

6 In fact, we do not even need that fact that the functor preserves composition, only that it preserves identity maps.

7 https://ncatlab.org/nlab/show/retract+argument

\section{References}

Altenkirch, T., Capriotti, P., Dijkstra, G., Kraus, N. and Forsberg, F. N. (2018). Quotient inductive-inductive types. In: FoSSaCS, Lecture Notes in Computer Science, vol. 10803, Springer, 293-310. https://doi.org/10.1007/978-3-31989366-2_16.

Altenkirch, T., Capriotti, P. and Kraus, N. (2016). Extending homotopy type theory with strict equality. In: CSL, LIPIcs, vol. 62, Schloss Dagstuhl - Leibniz-Zentrum fuer Informatik, 21:1-21:17. https://doi.org/10.4230/LIPIcs.CSL.2016.21.

Angiuli, C., Brunerie, G., Coquand, T., Hou, K.-B., Harper, R. and Licata, D. R. (2019). Cartesian cubical type theory. Draft. https:/github.com/dlicata335/cart-cube/blob/master/cart-cube.pdf.

Angiuli, C., Hou, K.-B. F. and Harper, R. (2018). Cartesian cubical computational type theory: Constructive reasoning with paths and equalities. In: Computer Science Logic 2018. https://doi.org/10.4230/LIPIcs.CSL.2018.6.

Bezem, M., Coquand, T. and Huber, S. (2013). A model of type theory in cubical sets. In: TYPES, LIPIcs, vol. 26, Schloss Dagstuhl - Leibniz-Zentrum fuer Informatik, 107-128. https://doi.org/10.4230/LIPIcs.TYPES.2013.107.

Bezem, M., Coquand, T. and Huber, S. (2019). The univalence axiom in cubical sets. The Journal of Automated Reasoning 63 (2) 159-171. https://doi.org/10.1007/s10817-018-9472-6.

Bezem, M., Coquand, T. and Parmann, E. (2015). Non-constructivity in Kan simplicial sets. In: Altenkirch, T. (ed.) 13th International Conference on Typed Lambda Calculi and Applications (TLCA 2015), Leibniz International Proceedings in Informatics (LIPIcs), vol. 38, Dagstuhl, Germany, Schloss Dagstuhl-Leibniz-Zentrum fuer Informatik, 92-106. ISBN 9783-939897-87-3. doi: 10.4230/LIPIcs.TLCA.2015.92. http://drops.dagstuhl.de/opus/volltexte/2015/5157.

Capriotti, P. (2017). Models of Type Theory with Strict Equality. Phd thesis, University of Nottingham, UK. http://eprints.nottingham.ac.uk/39382/.

Cavallo, E. and Harper, R. (2019). Higher inductive types in cubical computational type theory. PACMPL 3 (POPL) 1:1-1:27. https://doi.org/10.1145/3290314.

Cohen, C., Coquand, T., Huber, S. and Mörtberg, A. (2017). Cubical type theory: A constructive interpretation of the univalence axiom. FLAP 4 (10) 3127-3170. http://collegepublications.co.uk/ifcolog/?00019.

Coquand, T., Huber, S. and Mörtberg, A. (2018). On higher inductive types in cubical type theory. In: LICS, ACM, 255-264. https://doi.org/10.1145/3209108.3209197.

Gambino, N. and Garner, R. (2008). The identity type weak factorisation system. Theoretical Computer Science 409 (1) 94-109. https://doi.org/10.1145/3209108.3209197.

Gambino, N. and Sattler, C. (2015). Uniform fibrations and the frobenius condition. arXiv preprint arXiv:1510.00669. https://arxiv.org/abs/1510.00669.

Hirschhorn, P. S. (2003). Model Categories and Their Localizations. Mathematical Surveys and Monographs, vol. 99, American Mathematical Society, Providence, RI.

Hofmann, M. (1997). Syntax and semantics of dependent types. In: Extensional Constructs in Intensional Type Theory, Springer, 13-54.

Homotopy Type Theory wiki. (2014). Homotopy type system. (consulted 10/14/2019). https://ncatlab.org/ homotopytypetheory/show/Homotopy+Type+System.

Hovey, M. (2007). Model Categories, vol. 63, American Mathematical Society.

Kapulkin, C. and Lumsdaine, P. L. (2012). The simplicial model of univalent foundations (after voevodsky). arXiv preprint arXiv:1211.2851. https://arxiv.org/abs/1211.2851.

Licata, D. R., Orton, I., Pitts, A. M. and Spitters, B. (2018). Internal universes in models of homotopy type theory. In: FSCD, LIPIcs, vol. 108, Schloss Dagstuhl - Leibniz-Zentrum fuer Informatik, 22:1-22:17. https://doi.org/10.4230/ LIPIcs.FSCD.2018.22.

Lumsdaine, P. L. (2011). Model structures from higher inductive types. Unpublished note. http://peterlefanulumsdaine. com/research/Lumsdaine-Model-strux-from-HITs.pdf.

Nuyts, A. (2018). Robust notions of contextual fibrancy. In: Presentation at HoTT/UF'18 Workshop. https://hott-uf. github.io/2018/abstracts/HoTTUF18_paper_2.pdf. 
Orton, I. and Pitts, A. M. (2018a). Axioms for modelling cubical type theory in a topos. Logical Methods in Computer Science 14 (4). https://doi.org/10.23638/LMCS-14(4:23)2018.

Orton, I. and Pitts, A. M. (2018b). Code supporting Axioms for Modelling Cubical Type Theory in a Topos. https://doi.org/10.17863/CAM.21675.

Sattler, C. (2017). The Equivalence Extension Property and Model Structures. arXiv preprint arXiv:1704.06911. https://arxiv.org/abs/1704.06911.

Swan, A. W. (2016). An algebraic weak factorisation system on 01-substitution sets: A constructive proof. Journal of Logic \& Analysis 8. http://logicandanalysis.org/index.php/jla/article/view/274/109.

The Coq Development Team. (2018). The Coq Proof Assistant Reference Manual. http://coq.inria.fr. Version 8.9.

Voevodsky, V. (2013). A simple type system with two identity types. Unpublished note. https://www.math.ias.edu/ vladimir/sites/math.ias.edu.vladimir/files/HTS.pdf.

\section{Appendix A. Prop Universe for Martin-Löf type Theory}

$$
\begin{aligned}
& \frac{\Gamma \vdash A \quad \Gamma, x: A \vdash P: \mathbb{P}}{\Gamma \vdash \forall x: A . P: \mathbb{P}} \\
& \underline{\forall x: A . P} \simeq_{\beta \eta} \Pi x: A . \underline{P} \\
& \frac{\Gamma \vdash A \quad \Gamma, x: A \vdash P: \mathbb{P}}{\Gamma \vdash \exists x: A \cdot P: \mathbb{P}} \\
& \frac{\Gamma \vdash t: A \quad \Gamma \vdash u: \underline{P\{x:=t\}}}{\Gamma \vdash\langle t, u\rangle: \underline{\exists x: A . P}} \\
& \frac{\Gamma \vdash R: \mathbb{P} \quad \Gamma \vdash f: \Pi x: A . \underline{P} \rightarrow \underline{R}}{\Gamma \vdash \exists-\operatorname{elim}(R, f): \underline{\exists x: A . P} \rightarrow \underline{R}} \\
& \frac{\Gamma \vdash P, Q: \mathbb{P}}{\Gamma \vdash P \wedge Q: \mathbb{P}} \quad \frac{\Gamma \vdash t: \underline{P} \quad \Gamma \vdash u: \underline{Q}}{\Gamma \vdash(t, u): \underline{P \wedge Q}} \quad \frac{\Gamma \vdash t: \underline{P \wedge Q}}{\Gamma \vdash \pi_{1}(t): \underline{P}} \quad \frac{\Gamma \vdash t: \underline{P \wedge Q}}{\Gamma \vdash \pi_{2}(t): \underline{Q}} \quad \pi_{1}\left(t, t^{\prime}\right) \simeq_{\beta \eta} t \\
& \pi_{2}\left(t, t^{\prime}\right) \simeq_{\beta \eta} t^{\prime} \\
& \frac{\Gamma \vdash P, Q: \mathbb{P}}{\Gamma \vdash P \vee Q: \mathbb{P}} \quad \frac{\Gamma \vdash t: \underline{P}}{\Gamma \vdash \operatorname{inl} t: \underline{P \vee Q}} \quad \frac{\Gamma \vdash t: \underline{Q}}{\Gamma \vdash \operatorname{inr} t: \underline{P \vee Q}} \\
& \frac{\Gamma \vdash R: \mathbb{P} \quad \Gamma \vdash f: \underline{P} \rightarrow \underline{R} \quad \Gamma \vdash f: \underline{Q} \rightarrow \underline{R}}{\Gamma \vdash \vee-\operatorname{elim}(R, f, g): \underline{P \vee Q \rightarrow \underline{R}}} \\
& \vee-\operatorname{elim}(R, f, g)(\operatorname{inl} t) \simeq_{\beta \eta} f t \\
& \vee-\operatorname{elim}(R, f, g)(\operatorname{inr} t) \simeq_{\beta \eta} g t \\
& \frac{\Gamma \vdash}{\Gamma \vdash \perp: \mathbb{P}} \\
& \frac{\Gamma \vdash A}{\Gamma \vdash \perp-\operatorname{elim}: \perp \rightarrow A} \\
& \frac{\Gamma \vdash}{\Gamma \vdash \top: \mathbb{P}} \\
& \frac{\Gamma \vdash}{\Gamma \vdash t t: 工}
\end{aligned}
$$

Remark. The dependent eliminators for $\exists, \wedge$, and $\vee$ can be recovered with proof irrelevance.

The logical equivalence of two propositions is the proposition defined by:

$$
P \Leftrightarrow Q:=(\underline{P} \rightarrow Q) \wedge(\underline{Q} \rightarrow P) .
$$

\section{Appendix B. Inconsistency of Regular Fibrant Replacement [Inconsistency . v]}

We demonstrate here why a fibrant replacement for full fibrancy would be inconsistent. We replay for this the proof given on the nLab (Homotopy Type Theory wiki 2014), given there in the context of Homotopy Type System. 
Let us assume that for any (open) type, there is a fibrant replacement which is fully fibrant and let us derive a contradiction. In particular, for any fibrant family $x . P$, the family $x . \bar{P}$ if regularly fibrant:

$$
\frac{\Gamma, x: A \vdash P}{\operatorname{RFib}(x: A . \bar{P})}
$$

Let $i . P$ be the family over the interval given by:

$$
P:=\lambda i: \square . i \equiv 1
$$

The family $i$. $P$ has been chosen such that $P 0 \cong \perp$ and $P 1 \cong \top$. There is $\star:=\eta\left(\right.$ refl $\left._{\equiv} 1\right)$ an element of type $\overline{P 1}$. Because the family $i . \bar{P} i$ is regularly fibrant, we can transport $\star$ along the path between 1 and 0 and get an element of $\overline{P 0}$. But there is a map $\overline{P 0} \rightarrow \perp$ which is induced by the map $P 0 \rightarrow \perp$ ( $\perp$ is fibrant), hence a contradiction.

\section{Appendix C. Axioms of the Formalization}

The following is the (reordered) output of the Coq command Print Assumptions applied to the Theorem 25 and the characterizations of Proposition 27.

\section{Axioms about equality in Martin-Löf type theory}

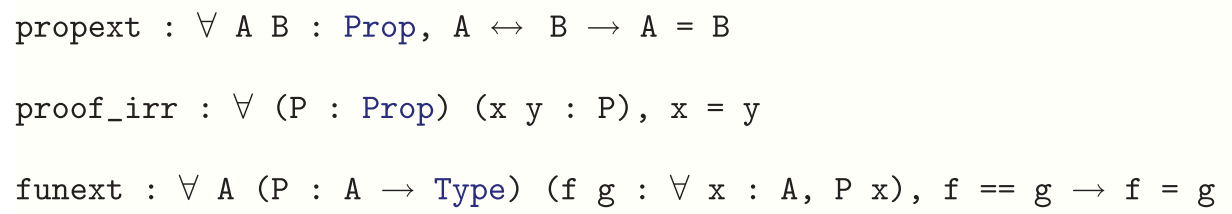

\section{Axioms about $\llbracket$ TT}

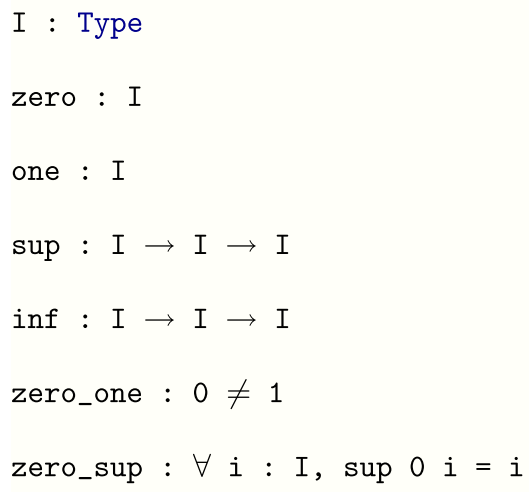




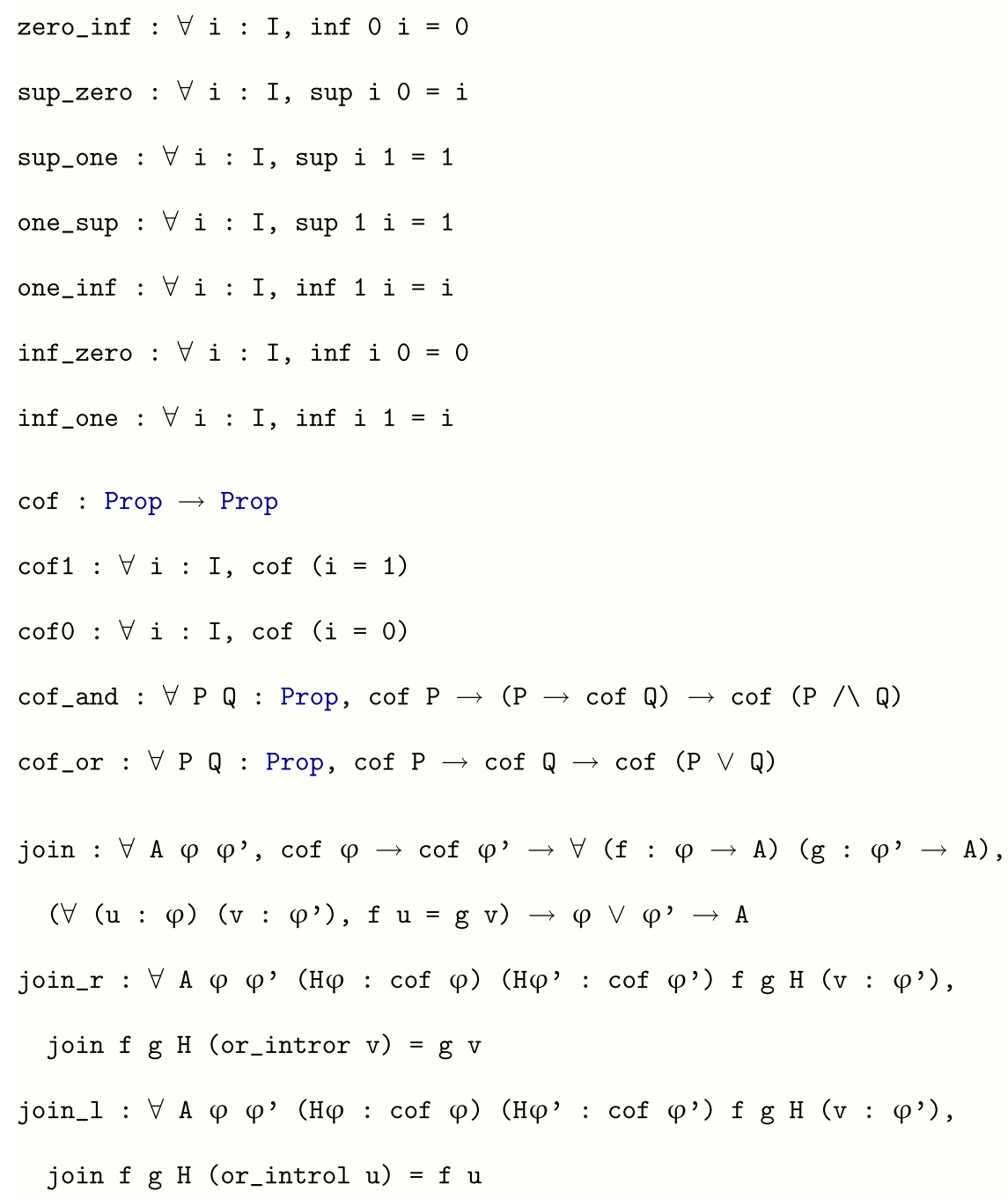

\section{Axioms about the QITs ( fibrant replacement and pushout)}

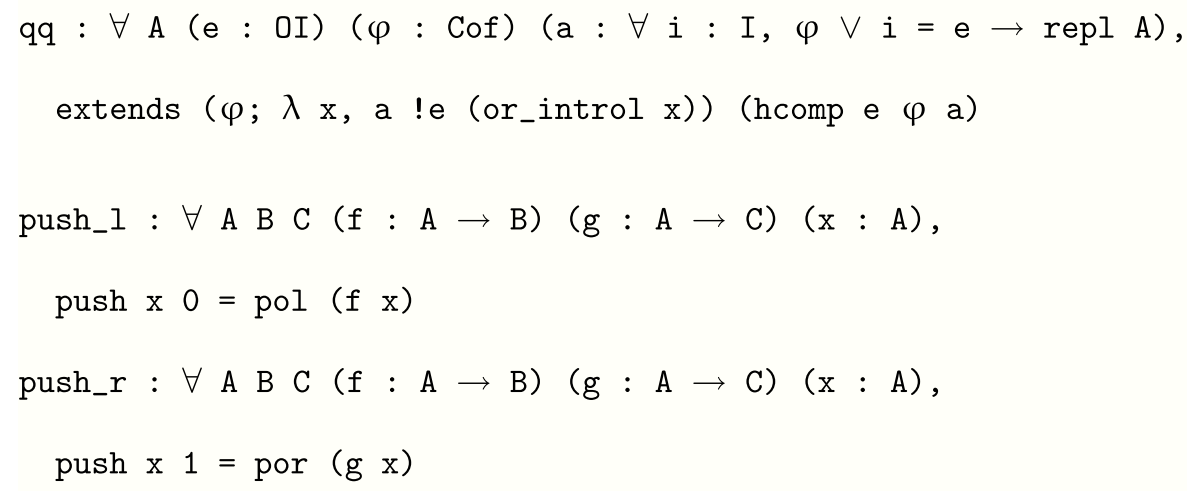




\section{Extension rule}

extension_rule__emptyctx $: \forall A(P: A \rightarrow$ Type $)$,

RFib $\mathrm{P} \rightarrow \exists$ lift $:$ repl $\mathrm{A} \rightarrow$ Type, RFib lift $\times \forall \mathrm{x}$, lift $(\eta \mathrm{x})==\mathrm{P} \mathrm{x}$

Remark. Let us remark that our formalization does not use connected $\left(\mathbf{a x}_{\mathbf{1}}\right)$, cof_ $\forall\left(\mathbf{a x}_{\mathbf{8}}\right)$, and strictness ( $\left.\mathbf{a x}_{9}\right)$ axioms. They are however hidden behind the extension rule. 